\title{
Development and testing of the Active Temperature, Ozone and Moisture Microwave Spectrometer (ATOMMS) cm and mm wavelength occultation instrument
}

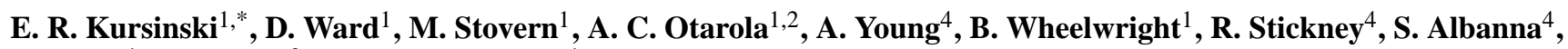 \\ B. Duffy ${ }^{4}$, C. Groppi ${ }^{3}$, and J. Hainsworth ${ }^{4}$ \\ ${ }^{1}$ University of Arizona, Department of Atmospheric Sciences, Tucson, AZ 85721, USA \\ ${ }^{2}$ TMT Observatory, Inc., Pasadena, CA 91125, USA \\ ${ }^{3}$ Arizona State University, School of Earth and Space Exploration, Tempe, AZ 85287, USA \\ ${ }^{4}$ University of Arizona, Department of Astronomy, Tucson, AZ 85721, USA \\ *now at: Broad Reach Engineering, Golden, CO 80401, USA
}

Correspondence to: E. R. Kursinski (rkursinski@broadreachengineering.com)

Received: 17 March 2011 - Published in Atmos. Meas. Tech. Discuss.: 22 July 2011

Revised: 24 January 2012 - Accepted: 1 February 2012 - Published: 27 February 2012

\begin{abstract}
We present initial results from testing a new remote sensing system called the Active Temperature, Ozone and Moisture Microwave Spectrometer (ATOMMS). ATOMMS is designed as a satellite-to-satellite occultation system for monitoring climate. We are developing the prototype instrument for an aircraft to aircraft occultation demonstration. Here we focus on field testing of the ATOMMS instrument, in particular the remote sensing of water by measuring the attenuation caused by the $22 \mathrm{GHz}$ and $183 \mathrm{GHz}$ water absorption lines.

Our measurements of the $183 \mathrm{GHz}$ line spectrum along an $820 \mathrm{~m}$ path revealed that the AM 6.2 spectroscopic model provdes a much better match to the observed spectrum than the MPM93 model. These comparisons also indicate that errors in the ATOMMS amplitude measurements are about $0.3 \%$. Pressure sensitivity bodes well for ATOMMS as a climate instrument. Comparisons with a hygrometer revealed consistency at the $0.05 \mathrm{mb}$ level, which is about $1 \%$ of the absolute humidity.

Initial measurements of absorption by the $22 \mathrm{GHz}$ line made along a $5.4 \mathrm{~km}$ path between two mountaintops captured a large increase in water vapor similar to that measured by several nearby hygrometers. A storm passage between the two instruments yielded our first measurements of extinction by rain and cloud droplets. Comparisons of ATOMMS $1.5 \mathrm{~mm}$ opacity measurements with measured visible opacity and backscatter from a weather radar revealed features simultaneously evident in all three datasets confirming the ATOMMS measurements. The combined ATOMMS, radar and visible information revealed the evolution of rain and
\end{abstract}

cloud amounts along the signal path during the passage of the storm. The derived average cloud water content reached typical continental cloud amounts. These results demonstrated a significant portion of the information content of ATOMMS and its ability to penetrate through clouds and rain which is critical to its all-weather, climate monitoring capability.

\section{Introduction and overview: the ATOMMS concept}

Reducing uncertainty about future climate change to support informed decision-making must be a key focus of climate research. Reducing uncertainty requires observations that determine how climate is actually changing, independent of models. This information is critical to assessing climate model realism. In addition, high precision, high resolution observations measuring variability are needed to tightly constrain the key processes and improve their representation in climate models in order to improve their forecasting skill.

The Active Temperature, Ozone and Moisture Microwave Spectrometer (ATOMMS) is a $\mathrm{cm}$ and $\mathrm{mm}$ wavelength remote sensing system we are developing to achieve unprecedented performance and attain key unfulfilled observational goals for measuring climate and reducing uncertainty about future climate (Kursinski et al., 2002, 2009).

Like GPS Radio Occultation (RO) (Kursinski et al., 1997; Anthes et al., 2011), ATOMMS is a satellite-to-satellite RO system (see Fig. 1). GPS RO is now the 4th most influential satellite system on Numerical Weather Prediction (NWP) (Cardinali, 2009) and was recommended by the Earth 


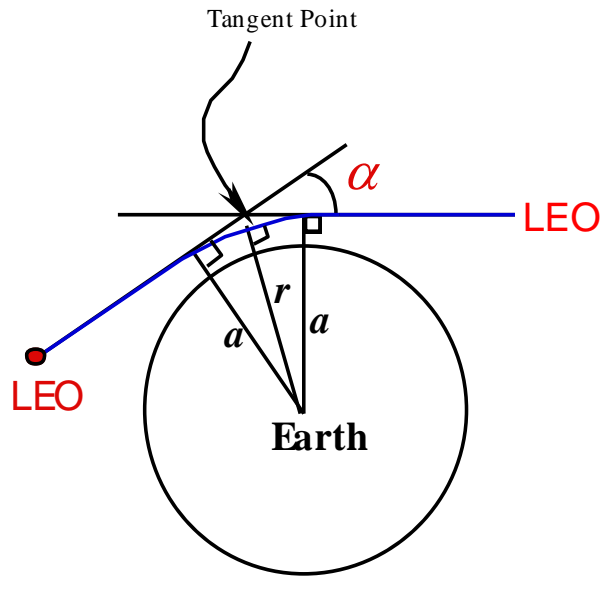

Fig. 1. ATOMMS occultation geometry.

Decadal Survey (Anthes et al., 2007) to become a core, longterm, operational observing system. As Fig. 2 summarizes, ATOMMS extends beyond the capabilities of GPS RO by using frequencies that probe absorption lines of key atmospheric constituents (unlike GPS whose frequencies were selected specifically to minimize interactions with the atmosphere). As a result, ATOMMS has the ability to retrieve the trace gas constituents profiled by NASA's Microwave Limb Sounder (MLS) (Waters et al., 2006) but with the much higher vertical resolution, precision, accuracy and allweather, global sampling of GPS RO. When implemented as a constellation of small spacecraft analogous to the present COSMIC GPS RO mission, ATOMMS will yield an unprecedented combination of performance by delivering measured atmospheric profiles that are unbiased by a priori model assumptions with complete global and diurnal coverage, which are critical for monitoring and understanding climate.

ATOMMS achieves its unique performance via differential absorption by measuring signal levels at two or more frequencies simultaneously in order to reduce or eliminate many types of common mode noise (Kursinski et al., 2002, 2004, 2009). The ATOMMS prototype instrument that we are developing at the University of Arizona probes atmospheric absorption within two spectral intervals near the $22 \mathrm{GHz}$ and $183 \mathrm{GHz}$ absorption lines of water vapor. The low-band portion of the instrument transmits and receives 8 monochromatic tones spaced approximately every $1 \mathrm{GHz}$ from 18.5 to $25.5 \mathrm{GHz}$ in order to simultaneously measure and separate the absorption spectra of water vapor and liquid water (Kursinski et al., 2009). The high-band portion presently transmits and receives two tones that are tunable over the interval between $182 \mathrm{GHz}$ and $205 \mathrm{GHz}$ that contains several absorption lines including $\mathrm{H}_{2} \mathrm{O}, \mathrm{O}_{3}, \mathrm{~N}_{2} \mathrm{O}$ and $\mathrm{H}_{2}^{18} \mathrm{O}$. We will be upgrading the high band system in the near future to measure signals at four frequencies simultaneously. The present instrument is being developed for an aircraft-toaircraft demonstration of the ATOMMS occultation concept.

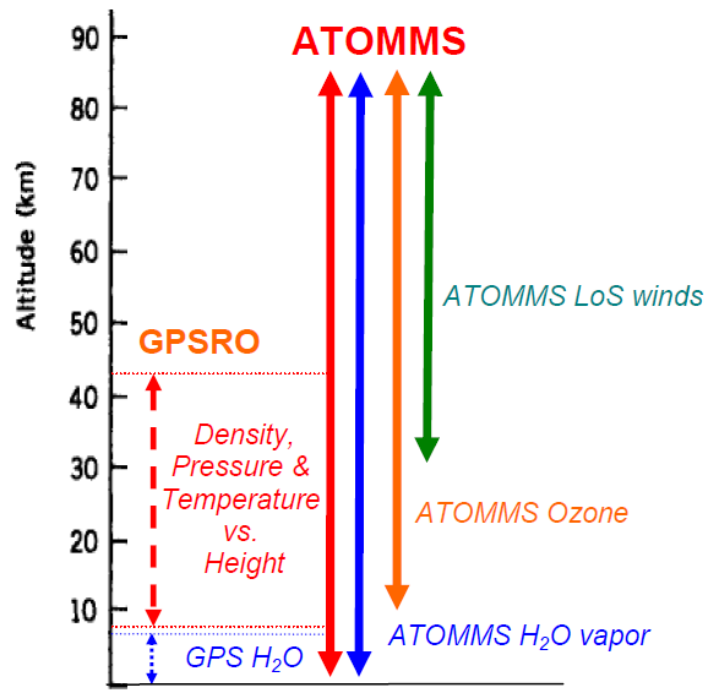

Fig. 2. Altitude comparison of ATOMMS vs. GPSRO. GPS profiles atmospheric density, pressure and temperature between the $230 \mathrm{~K}$ altitude in the troposphere (where water vapor begins contributing significantly to refractivity) to approximately $45 \mathrm{~km}$, depending on electron densities in the ionosphere. At tropospheric temperatures warmer than $240 \mathrm{~K}$, GPS refractivity primarily constrains water vapor. In contrast, ATOMMS simultaneously profiles density, pressure, temperature and water vapor from the lower troposphere to the mesopause. ATOMMS profiles ozone from the upper troposphere to the mesopause. Above the $\sim 10 \mathrm{mb}$ level, ATOMMS will also determine line of sight $(\mathrm{LoS})$ winds via the Doppler shift of the center of absorption lines.

Here we present some results from the ground based testing of the prototype ATOMMS instrument to date.

\section{$2183 \mathrm{GHz}$ spectra along $820 \mathrm{~m}$ path}

In this section we discuss ATOMMS measurements taken during ground testing of the "high band" (182-205 GHz) portion of the instrument on 13 March 2010. In these tests, signals were transmitted across the University of Arizona campus over an $820 \mathrm{~m}$ path from the 8th floor rooftop of the Aerospace and Mechanical Engineering (AME) building to the 11th floor rooftop of the Gould-Simpson building (see Fig. 3). We used these measurements to track changes in water vapor content along the path over the $3.5 \mathrm{~h}$ duration of the test.

\subsection{Method}

In order to recover water vapor, ATOMMS measures the attenuation of the signal along its propagation path through the atmosphere. This is related to the atmospheric optical depth via Eq. (1). Because ATOMMS uses coherent signals, 


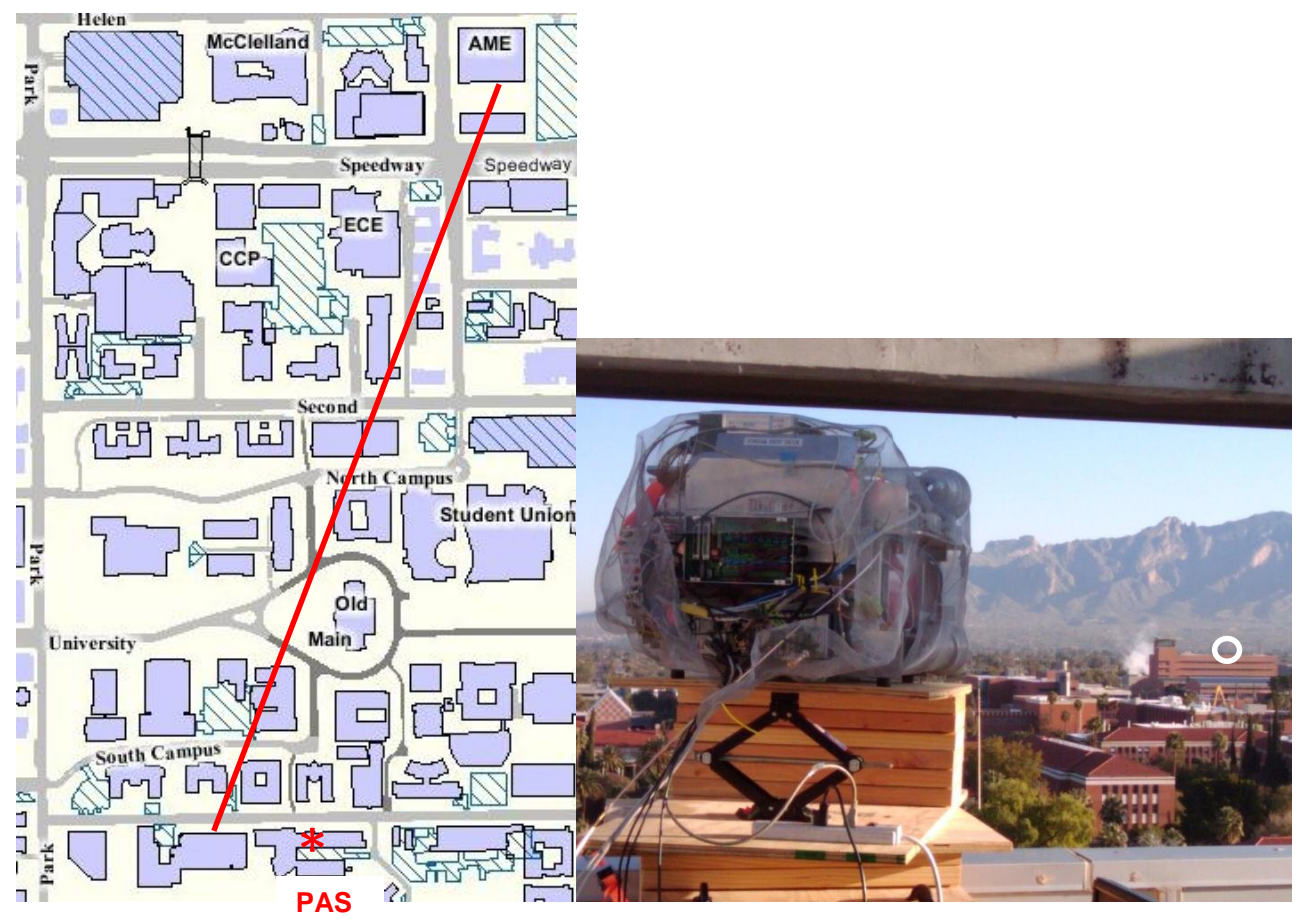

Fig. 3. Left panel: map of University of Arizona showing the ATOMMS rooftop to rooftop geometry. Path length is $820 \mathrm{~m}$. The red asterisk shows location of the hygrometer on the PAS building against which we compared the ATOMMS measurements. Right panel: ATOMMS B instrument looking toward ATOMMS A instrument on roof of AME building indicated by white circle. In the background are the Catalina Mountains where subsequent tests discussed below took place.

we write the absorption in terms of amplitude (rather than intensity)

$A=A_{0} e^{-\tau / 2}$

where $A$ is the signal amplitude after the absorption, $A_{0}$, the amplitude of the signal in the absence of atmospheric attenuation and $\tau$ is the optical depth. The optical depth, which is proportional to the water vapor along the path, is therefore given as

$\tau=2 \ln \left(\frac{A_{0}}{A}\right)$.

Thus, to determine the optical depth, we must determine $A$ and $A_{0}$.

As noted, ATOMMS is a differential absorption system that measures signal levels at two (or more) frequencies simultaneously in order to reduce or eliminate many types of common mode noise. The relationship between the amplitudes and the optical depth difference is

$\tau_{2}-\tau_{1}=2 \ln \left(\frac{A\left(f_{1}\right)}{A_{0}\left(f_{1}\right)} \frac{A_{0}\left(f_{2}\right)}{A\left(f_{2}\right)}\right)=F(q, T, P)$

where $q$ is the specific humidity, $P$ is the total pressure, $T$ is atmospheric temperature, and $f_{1}$ and $f_{2}$ are frequencies near the absorption line. Note that $q, T$ and $P$ in Eq. (3) represent path-averaged values, which is consistent with our subsequent data processing. For precise calculations, the optical depth is a path-integrated quantity and explicitly depends of the variation of $q, T$, and $P$ along the propagation path. In the satellite-to-satellite geometry, $A_{0}\left(f_{1}\right)$ and $A_{0}\left(f_{2}\right)$ will be determined by measurements immediately before or after each occultation when the lowest point along the signal path between the spacecraft is several hundred $\mathrm{km}$ above the altitudes in the atmosphere that are detectable by ATOMMS. For rooftop measurements, determining $A_{0}\left(f_{1}\right)$ and $A_{0}\left(f_{2}\right)$ is more difficult because we cannot remove the atmosphere between the transmitter and receiver. Thus, in this early ATOMMS testing, we have been unable to calibrate our observations relative to a vacuum and retrieve the absolute amount of water vapor along the path. However, we have been able to apply a differential approach to accurately measure changes in water vapor with time by ratioing the amplitudes measured at each time to the amplitudes measured at some reference time.

We had planned to measure the ratio of $A_{0}\left(f_{1}\right)$ to $A_{0}\left(f_{2}\right)$ in an anechoic chamber to effectively determine the instrument response in a vacuum that would allow us to determine the absolute water vapor amount. However, our measurements taken out in the field are affected by local multipath where the ATOMMS signals take not only the direct straight line signal path between the transmitter and the receiver but also at least one secondary path where the signal reflects off 
some object on its way to the receiver. The signals from the multiple paths then sum and interfere at the receiver which causes the signal amplitude to differ from that of the signal along just the direct path.

Our solution in the testing done to date is to hold the viewing geometry fixed and ratio the ATOMMS amplitude measured at each time with the ATOMMS amplitudes measured at a reference time, typically taken to be the time when the minimum water vapor was measured. By holding the geometry fixed, the multipath effect remains constant and common to all measurements and therefore divides out when the amplitude ratio is formed such that the ratio yields a clean differential absorption spectrum. We have used this approach to achieve the results presented below.

We define the optical depth difference as

$\Delta \tau\left(f, f_{\mathrm{ref}} ; q, P, T\right)=\tau(f ; q, P, T)-\tau\left(f_{\mathrm{ref}} ; q, P, T\right)$

where $f$ is a frequency on the absorption line and $f_{\text {ref }}$ is a frequency selected to be farther from the line center where there is relatively little absorption. For a given pressure and temperature, the optical depth difference for a given frequency and reference frequency varies approximately linearly with the integrated water vapor along the path (except for a small residual dry continuum).

$$
\begin{aligned}
& \Delta \tau\left(f, f_{\mathrm{ref}} ; q, P, T\right)=q a(f ; P, T) \\
& \quad+\tau_{\text {dry }}(f ; P, T)-q b\left(f_{\text {ref }} ; P, T\right)-\tau_{\text {dry }}\left(f_{\text {ref }} ; P, T\right) .
\end{aligned}
$$

The $a$ and $b$ terms include both the absorption line and the wet continuum. $\tau_{\text {dry }}$ represents the dry continuum, which is small and varies slowly with frequency. Since the fractional difference between $f$ and $f_{\text {ref }}$ over the range of the ATOMMS "high band" frequencies is less than $10 \%$, the two dry continuum terms largely cancel out. So

$\Delta \tau\left(f, f_{\mathrm{ref}} ; q, P, T\right) \cong q\left[a(f ; P, T)-b\left(f_{\mathrm{ref}} ; P, T\right)\right]$.

The next step is to normalize each optical depth difference, $\Delta \tau\left(f, f_{\mathrm{ref}} ; q(t), P(t), T(t)\right)$, measured at time, $t$, by ratioing it to the optical depth difference measured at a chosen normalization time $t_{0}, \Delta \tau_{\text {norm }}\left(f, f_{\text {ref }} ; q\left(t_{0}\right), P\left(t_{0}\right), T\left(t_{0}\right)\right)$ observed during the test.

$$
\begin{aligned}
& \frac{\Delta \tau\left(f, f_{\mathrm{ref}} ; q(t), P(t), T(t)\right)}{\Delta \tau\left(f, f_{\mathrm{ref}} ; q_{\mathrm{norm}}\left(t_{0}\right), P\left(t_{0}\right), T\left(t_{0}\right)\right)} \\
= & \frac{q(t)\left[a(f ; P(t), T(t))-b\left(f_{\mathrm{ref}} ; P(t), T(t)\right)\right]}{q_{\mathrm{norm}}\left(t_{0}\right)\left[a\left(f ; P\left(t_{0}\right), T\left(t_{0}\right)\right)-b\left(f_{\mathrm{ref}} ; P\left(t_{0}\right), T\left(t_{0}\right)\right)\right]}
\end{aligned}
$$

where $q_{\text {norm }}\left(t_{0}\right)$ is the specific humidity at time $t_{0}$, which is used as the specific humidity normalization. Therefore as long as the pressure and temperature do not vary much from $t_{0}$ to $t$, as was the case for our rooftop measurements (see Sect. 2.2), we can write

$$
\frac{\Delta \tau\left(f, f_{\mathrm{ref}} ; q(t), P(t), T(t)\right)}{\Delta \tau\left(f, f_{\mathrm{ref}} ; q\left(t_{0}\right), P\left(t_{0}\right), T\left(t_{0}\right)\right)} \cong \frac{q(t)}{q_{\mathrm{norm}}\left(t_{0}\right)} .
$$

So the ratio of the optical depth differences at two times approximately equals the ratio of the path integrated water at those two times.
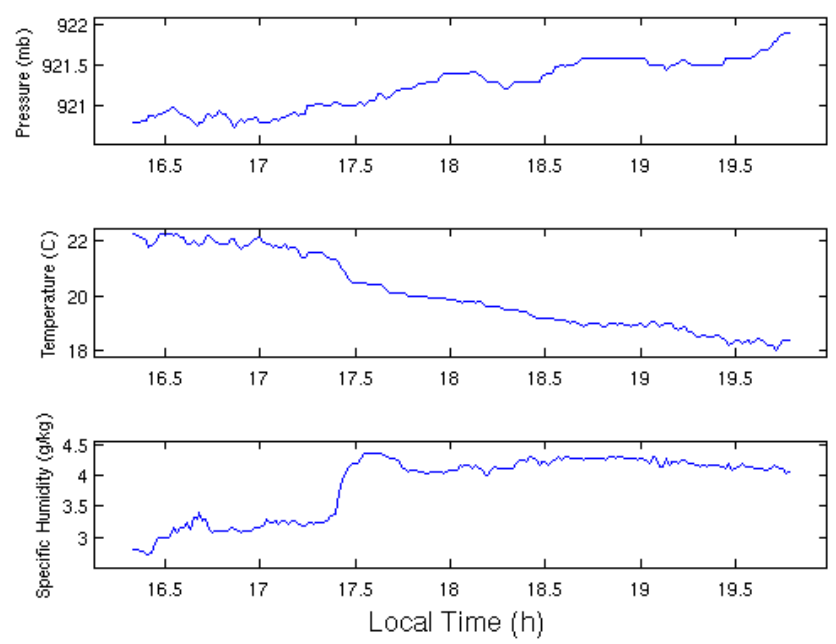

Fig. 4. The atmospheric pressure, temperature and specific humidity measured at the PAS building during the ATOMMS rooftop tests on 13 March 2010.

\subsection{Results}

For the tests performed on 13 March 2010, our best observations were obtained when the reference frequency was set to $200.6 \mathrm{GHz}$, while the other transmit frequency was tuned over the range from $183.60 \mathrm{GHz}$ to $187.50 \mathrm{GHz}$ in steps of $0.15 \mathrm{GHz}$ for a total of 27 frequencies. Each individual tuned frequency was held for one second and thus it took $27 \mathrm{~s}$ to tune through this frequency range. This cycle of observations was repeated every $224 \mathrm{~s}$ during the $3.5 \mathrm{~h}$ duration of the test, which allowed us to make 56 estimates of the change in vapor pressure relative to a chosen normalization time, with a time spacing of $224 \mathrm{~s}$. We also note that the ATOMMS measurements are inherently quite fast and ATOMMS will be able to determine water at least two orders of magnitude faster than the approximately once per $224 \mathrm{~s}$ sampling shown in the figures. This sampling interval was an artifact of the particular test configuration run on this day. The ATOMMS instrument will estimate water vapor at $1 \mathrm{~s}$ or shorter intervals.

Figure 4 shows the air pressure, temperature, and specific humidity measured during this test by sensors on the roof of the Physics and Atmospheric Sciences (PAS) Building, which is located next door to the building where the ATOMMS receiver was operating. In the results shown below, we utilized the first set of ATOMMS measurements, centered at $16.35 \mathrm{~h}$, as the normalization time. The corresponding specific humidity from Fig. 4 is $2.80 \mathrm{~g} \mathrm{~kg}^{-1}$. The maximum measured specific humidity was $4.34 \mathrm{~g} \mathrm{~kg}^{-1}$ at $17.58 \mathrm{~h}$, which corresponds closest in time with the $21 \mathrm{st}$ set of ATOMMS observations. Equation (8) is valid for all frequency pairs $\left(f ; f_{\text {ref }}\right)$, thus we can form the ratio of the amplitude spectra at these selected times and water vapor amounts 


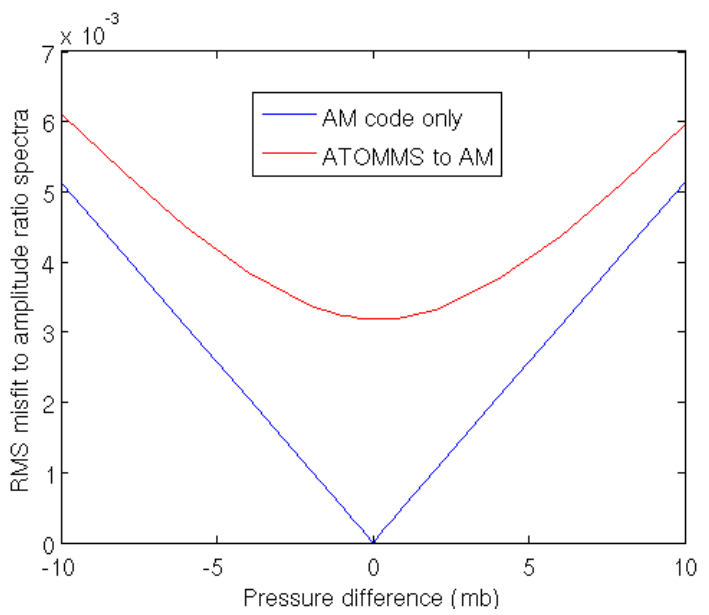

Fig. 5. Sensitivity of ATOMMS $183 \mathrm{GHz}$ spectra defined in Eq. (9) to variations in pressure. The blue line is the RMS difference between the AM 6.2 model and itself as the pressure difference is varied. The red line is the difference between the ATOMMS spectra and the AM 6.2 model The minimum error occurs about $0.1 \mathrm{mb}$ above the measured surface pressure.

$$
\begin{aligned}
& \frac{A\left(f ; f_{\mathrm{ref}} ; q_{\mathrm{max}}\left(t_{1}\right), P\left(t_{1}\right), T\left(t_{1}\right)\right)}{A\left(f ; f_{\mathrm{ref}} ; q_{\mathrm{norm}}\left(t_{0}\right), P\left(t_{0}\right), T\left(t_{0}\right)\right)} \\
& \quad=A_{\mathrm{SR}}\left(f ; f_{\mathrm{ref}} ; q_{\max }-q_{\mathrm{norm}}, P, T\right)
\end{aligned}
$$

where $A_{\mathrm{SR}}$ is the ratio of two spectra observed at different times and $t_{1}$ represents the time when the maximum specific humidity was observed. We made the approximations of replacing $P\left(t_{1}\right), P\left(t_{0}\right), T\left(t_{1}\right)$ and $T\left(t_{0}\right)$ with $P$ and $T$, which represent the path-averaged pressure and temperature.

As shown in Fig. 4, the peak to peak pressure variations during the test were about $\pm 0.5 \mathrm{mb}$ relative to a mean value of $921.4 \mathrm{mb}$ for a peak to peak variation of $\pm 0.05 \%$. As Fig. 5 shows, pressure variations this small will have negligible impact on the retrieval of changes in specific humidity. Peak to peak temperature variations were about $\pm 2{ }^{\circ} \mathrm{C}$ out of $293 \mathrm{~K}$ amounting to $\pm 0.7 \%$. Since the line width scales as $P / T^{0.77}$ (Payne et al., 2008), a fractional temperature error produces 0.77 times the impact of the fractional pressure error of the same magnitude. Therefore a $2{ }^{\circ} \mathrm{C}$ or $0.7 \%$ error in temperature is equivalent to a fractional pressure error of $0.5 \%$ or about $5 \mathrm{mb}$. According to Fig. 5 , the resulting error in amplitude ratio is $0.25 \%$ which is again very small. Therefore the resulting variations in the lineshape due to pressure and temperature essentially cancel in the ratio in Eq. (7) such that Eqs. (8) and (9) are applicable to these test conditions.

Equation (9) shows that the ratio of the two spectra is another spectrum that is proportional to the change in specific humidity, $q_{\max }-q_{\text {norm}}$. A series of these amplitude ratio spectra can be calculated from the measured ATOMMS amplitudes. Using the corresponding measurements of pressure and temperature, we used a microwave propagation model to determine the value of $q_{\max }-q_{\text {norm }}$ that best fits each

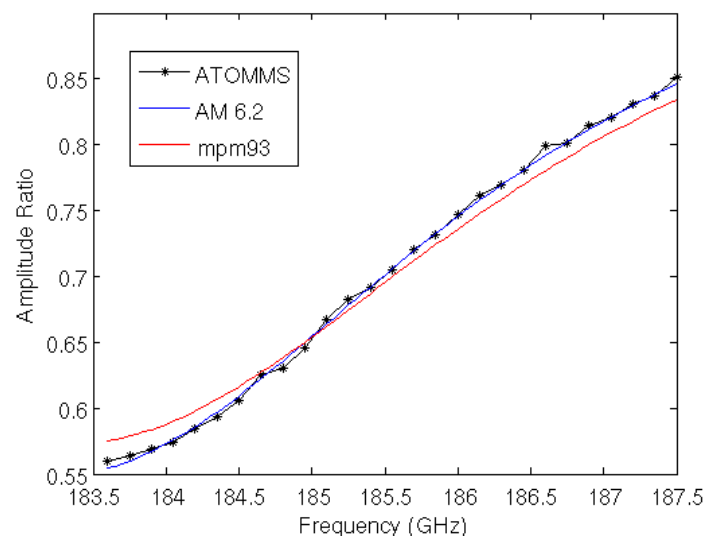

Fig. 6. Amplitude ratio as defined in Eq. (9) based on ATOMMS measurements of amplitude compared with the best fit value for $q_{\text {max }}-q_{\text {norm }}$ to the ATOMMS amplitude ratio as computed by two microwave propagation models, AM 6.2 and MPM93.

$183.60 \mathrm{GHz}$ to $187.50 \mathrm{GHz}$ amplitude ratio spectrum, $A_{\mathrm{SR}}$, obtained every $224 \mathrm{~s}$ from ATOMMS observations of amplitude. A comparison between the ATOMMS observations and the best fit results for the AM 6.2 model (Paine et al., 2011) and the MPM93 model (Liebe et al., 1993) is shown in Fig. 6.

Figure 6 shows that ATOMMS is useful in evaluating lineshape functions used in spectroscopic models. Specifically the figure shows that the AM 6.2 model gives a better fit to the measured lineshape than the MPM93 model indicating that the AM 6.2 model is a significantly more accurate spectroscopic model. The $q_{\max }-q_{\text {norm }}$ measured by the hygrometer on PAS was $1.51 \mathrm{~g} \mathrm{~kg}^{-1}$. The $q_{\max }-q_{\text {norm }}$ derived as the best fit of the ATOMMS measurements to the AM 6.2 model spectrum was $1.51 \mathrm{~g} \mathrm{~kg}^{-1}$, while that derived from the best fit using the MPM93 model yielded a somewhat lower change of $1.45 \mathrm{~g} \mathrm{~kg}^{-1}$. Since the AM 6.2 model provides a significantly better fit to the ATOMMS spectra, we use it for the rest of the results from this test.

We can apply the spectra-fitting algorithm described above at all of the 56 sets of observed ATOMMS spectra. To simplify the calculations, we used an average value for pressure of $921.4 \mathrm{mb}$ and an average value for temperature of $20^{\circ} \mathrm{C}$ for all of the times. As stated above the variations of pressure and temperature over the time of the ATOMMS measurements were quite small and have little effect on the best fit values for changes in specific humidity. The results are shown in Fig. 7. The ATOMMS-derived changes in specific humidity track the changes measured by the PAS hygrometer including the rapid increase in water vapor near $17.4 \mathrm{~h}$. It is also worth noting that the two measurement systems use entirely different physical principles to measure water vapor. The PAS hygrometer measures changes in capacitance caused by diffusion of moisture into and out of the dielectric material in a capacitor, while ATOMMS is a mmwavelength differential absorption spectrometer. One must 


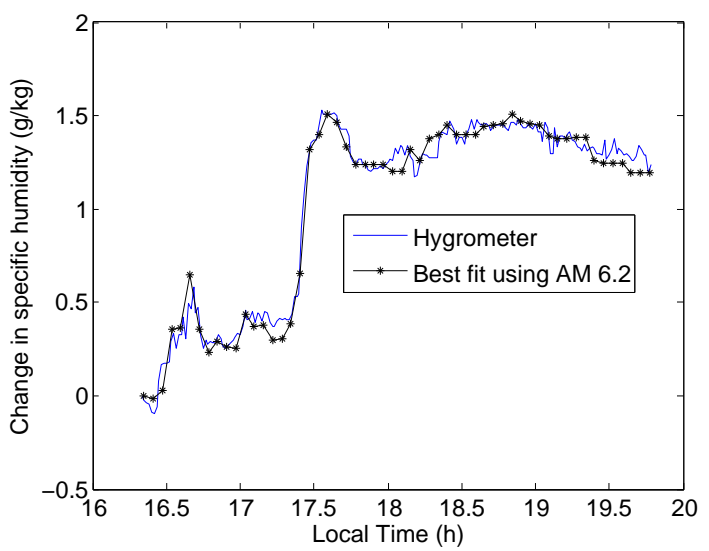

Fig. 7. Change in specific humidity relative to first observation. The black asterisks represent the best fit changes in specific humidity along the signal path to the ATOMMS amplitude ratio spectra each time an ATOMMS amplitude spectra was measured. The blue curve shows the changes in specific humidity as measured by the nearby hygrometer on the PAS building.

also remember that an exact match is not expected because the PAS hygrometer is a point measurement while ATOMMS is measuring changes in specific humidity integrated along an $820 \mathrm{~m}$ path.

During these measurements we encountered some interference when the receiver intermediate frequencies were below $5 \mathrm{GHz}$ that did not allow us to obtain good measurements over the entire ATOMMS frequency range for this test. Still better results would be expected if we had amplitude measurements over the entire ATOMMS "high band" frequency range of $182 \mathrm{GHz}-205 \mathrm{GHz}$.

Figure 5 was constructed to show the sensitivity of the amplitude spectral ratio, $A_{\mathrm{SR}}$, to changes in the path-averaged pressure along the propagation path. The blue curve shows how the RMS of the differences at the 27 measured ATOMMS frequencies across the range from $183.60 \mathrm{GHz}$ to $187.50 \mathrm{GHz}$ grows as the pressure is moved from its nominal value based on AM model calculations. The red curve shows the pressure sensitivity of the ATOMMS measurements via comparison with the AM model lineshape. The minimum discrepancy occurs about $0.1 \mathrm{mb}$ above the measured pressure. Errors of about $2 \mathrm{mb}$ are distinguishable for this particular set of observations, which corresponds to a fractional pressure uncertainty of about $0.2 \%$. If we assume that the blue curve represents the error due to pressure and that the differences between the blue and red curves are mainly due to ATOMMS measurement errors and turbulence, Fig. 5 also implies that the maximum RMS errors in the measured ATOMMS amplitude spectral ratios defined in Eq. (9) are about $0.3 \%$. This value is obtained by noting that at any given pressure difference in Fig. 5, the value of the red curve can be obtained by adding roughly a $0.3 \%$ error to the blue curve in a root sum squares sense. This ignores possible errors in the spectroscopic model.
The ability to measure line shape is a key advantage of ATOMMS over GPS RO because it enables ATOMMS to determine the upper boundary of the hydrostatic integral directly from the ATOMMS observations (Kursinski et al., 2002, 2009) without relying on an NWP analysis or climatology to set the upper boundary as GPS RO must do. This GPS RO sensitivity to systematic biases in those analyses limits the utility of GPS RO for climate in the mid stratosphere and above (e.g., Ho et al., 2009).

\section{Mountaintop observations near $22 \mathrm{GHz}$ on 20 August 2010}

\subsection{Background}

Because the $820 \mathrm{~m}$ distance used on campus was too short to achieve significant optical depths at $22 \mathrm{GHz}$, we designed tests to run between Mt. Bigelow $(2515 \mathrm{~m})$ and Mt. Lemmon ridge $(2752 \mathrm{~m})$ separated by approximately $5.4 \mathrm{~km}$ just north of the University of Arizona (Fig. 8a). On 20-21 August 2010 we ran our first ATOMMS mountaintop test. For cross comparisons we used three hygrometers located near the $5.4 \mathrm{~km}$ test path: the weather station in the town of Summerhaven at $2401 \mathrm{~m}$ (Fig. 8a); the Sustainability of semiArid Hydrology and Riparian Areas (SAHRA) eddy correlation tower on Mt. Bigelow approximately $400 \mathrm{~m}$ to the east of the ATOMMS instrument on Mt. Bigelow at an elevation of $2613 \mathrm{~m}$; and a Buck Instruments CR4 laboratory-quality chilled mirror hygrometer located at the site of the $22 \mathrm{GHz}$ transmitter on Mt. Bigelow at $2515 \mathrm{~m}$. These three hygrometers provide point measurements at different elevations in the vicinity of the test path. Figure 8b shows the ATOMMS $22 \mathrm{GHz}$ transmitter on Mt. Lemmon looking towards the receiver located at the observatory on Mt. Bigelow.

We recorded an 11.25 h data set from 21:45 until 09:00 LT (local time) the following day during which a plume of moisture advected into the greater Tucson area increasing water vapor concentrations by a factor of two. Figure 9 shows the moisture measured by the three hygrometers with each observing an overall rise in specific humidity over the test period. The Summerhaven hygrometer which is located near the point identified as "Summerhaven" in Fig. 8a, begins with the largest water vapor concentration and observes the smallest change in water vapor of the three hygrometers. We suspect this is associated with its location in the valley and a nocturnal thermal inversion that isolates it from larger changes in moisture at higher altitudes overnight and maintains a high moisture concentration via evapotranspiration at the surface which together hold moisture concentrations at the valley surface higher than those aloft. The two Bigelow hygrometers track one another more closely than the Summerhaven hygrometer through 26:00 including the rapid variations in humidity. After 27:00, the rapid variations in the Bigelow measurements are smaller and the Summerhaven 


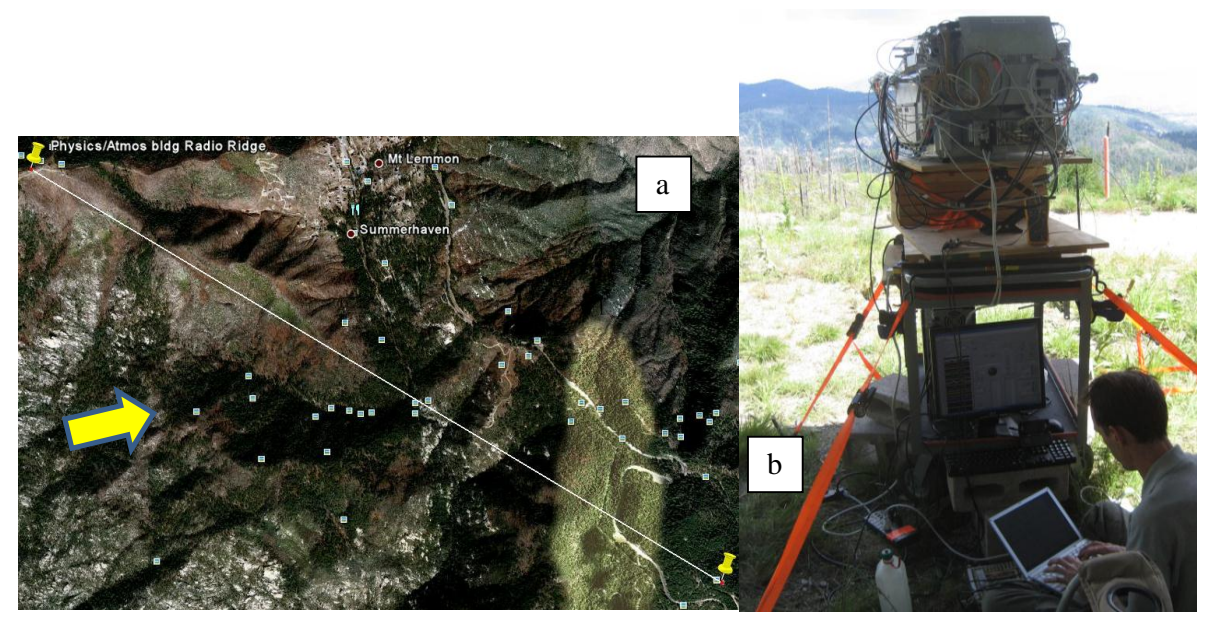

Fig. 8. The Mt. Lemmon (a) to Mt. Bigelow (b) geometry. (b) Abe Young at the ATOMMS-A instrument on Mt. Lemmon. The white dome in the distance in the upper left corner of the picture is the Mt. Bigelow observatory where the ATOMMS-B instrument is located. Yellow arrow indicates the approximate direction of the propagation of the storm on 28 August 2010.
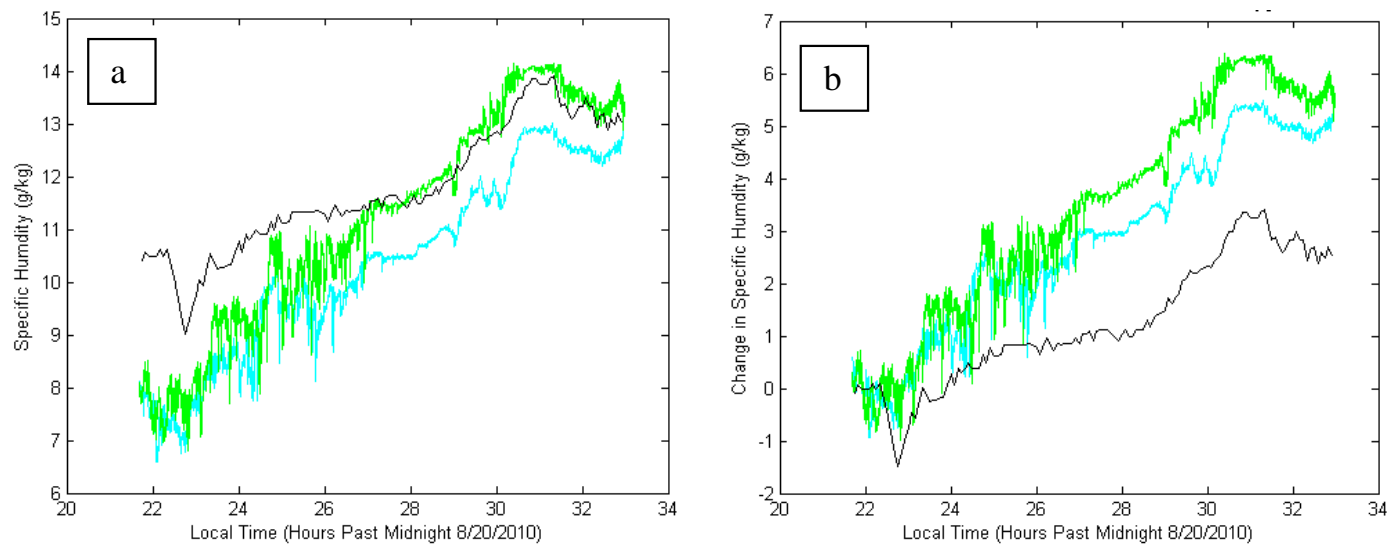

Fig. 9. Specific Humidity measurements during the test from hygrometers at Summerhaven (black), the SAHRA flux tower on Mt. Bigelow (green) and our chilled mirror hygrometer (cyan) at ATOMMS-B on Mt. Bigelow. (a) Specific humidity. (b) Change in specific humidity relative to beginning of test at 21:45 LT.

and SAHRA hygrometers have become quite similar while the chilled mirror moisture concentrations are about $10 \%$ lower than the other two. The SAHRA station observed the largest increase in moisture overnight. Figure $9 \mathrm{~b}$ indicates that the change in specific humidity relative to the first $30 \mathrm{~min}$ of the test was greater at higher altitudes.

\section{$3.222 \mathrm{GHz}$ retrievals}

The amplitudes of the signals measured by ATOMMS $22 \mathrm{GHz}$ receiver have been reduced by absorption due to the atmospheric water vapor along the path between the two instruments according to Eq. (1). To isolate the optical depth, via Eq. (2), we must know both $A$ and $A_{0}$. As in the campus tests, because we cannot determine the $A_{0}$ term, we determine changes in water vapor using the differential approach in time.
In order to solve for the change in specific humidity, $q$, relative to the specific humidity at the beginning of the test, $q(t)-q\left(t_{0}\right)$, we must solve for differential optical depth, $\Delta \tau$, at a particular frequency, $f$. By taking the ratio of two received amplitudes at different times, $t$ and $t_{0}$, we can then solve for the change in optical depth.

$\frac{A(t)}{A\left(t_{0}\right)} \frac{A_{0}\left(t_{0}\right)}{A_{0}(t)}=e^{\left(\frac{\tau\left(t_{0}\right)-\tau(t)}{2}\right)}$

To estimate the change in optical depth, we assume that the transmitter is stable over the time period of the two observations such that the $A_{0}$ terms cancel.

$\Delta \tau\left(t ; t_{0}\right) \cong-2 \ln \left(\frac{A(t)}{A\left(t_{0}\right)}\right)$.

Converting the change in optical depth over time to a change in water vapor concentration and specific humidity requires 
Table 1. Conversions between changes in optical depth and the average specific humidity, $\bar{q}$, along a $5.4 \mathrm{~km}$ path.

\begin{tabular}{lrrrrrr}
\hline AM6.2 Model & $\begin{array}{r}\bar{q}-1 \\
\left(\mathrm{~g} \mathrm{~kg}^{-1}\right)\end{array}$ & $\begin{array}{r}\bar{q} \\
\left(\mathrm{~g} \mathrm{~kg}^{-1}\right)\end{array}$ & $\begin{array}{r}\bar{q}+1 \\
\left(\mathrm{~g} \mathrm{~kg}^{-1}\right)\end{array}$ & $\begin{array}{l}K=[\mathrm{d} \tau] / \mathrm{d} \bar{q} \\
{\left[\mathrm{~g} \mathrm{~kg}^{-1}\right]^{-1}}\end{array}$ & $\begin{array}{l}\mathrm{d} \ln \bar{K} / \mathrm{d} P \\
(\% / \mathrm{mb})\end{array}$ & $\begin{array}{r}\mathrm{d} \ln \bar{K} / \mathrm{d} T \\
\left(\% /{ }^{\circ} \mathrm{C}\right)\end{array}$ \\
\hline Vapor pressure (mb) & 11.25 & 12.43 & 13.61 & & & \\
22.6 GHz Tau [nepers] & 0.359 & 0.394 & 0.428 & 0.0346 & -0.105 & -0.25 \\
23.5 GHz Tau [nepers] & 0.302 & 0.331 & 0.359 & 0.0285 & -0.21 & -0.56 \\
\hline & $\bar{q}-1$ & $\bar{q}$ & $\bar{q}+1$ & $K=[\mathrm{d} \tau] / \mathrm{d} \bar{q}$ & $\mathrm{~d} \ln \bar{K} / \mathrm{d} P$ & $\mathrm{dln} \bar{K} / \mathrm{d} T$ \\
MPM93 Model & $\left(\mathrm{g} \mathrm{kg}^{-1}\right)$ & $\left(\mathrm{g} \mathrm{kg}^{-1}\right)$ & $\left(\mathrm{g} \mathrm{kg}^{-1}\right)$ & {$\left[\mathrm{g} \mathrm{kg}^{-1}\right]^{-1}$} & $(\% / \mathrm{mb})$ & $\left(\% /{ }^{\circ} \mathrm{C}\right)$ \\
\hline Vapor pressure (mb) & 11.25 & 12.43 & 13.61 & & & \\
22.6 GHz Tau [nepers] & 0.322 & 0.354 & 0.385 & 0.0312 & -0.086 & -0.43 \\
23.5 GHz Tau [nepers] & 0.285 & 0.314 & 0.342 & 0.0287 & -0.049 & -0.54 \\
\hline
\end{tabular}

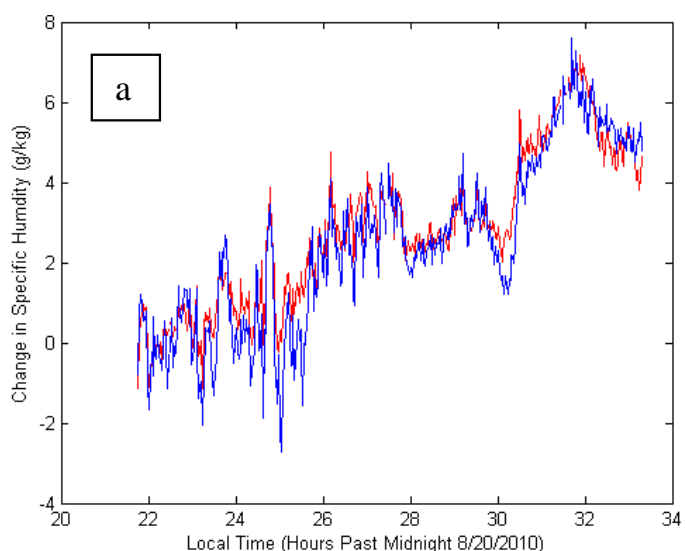

Fig. 10. (a) Conversion to specific humidity using the AM model $22.6 \mathrm{GHz}$ and $23.5 \mathrm{GHz}$ channels are red and blue respectively.

a spectroscopic model. Using the AM 6.2 model and the MPM93 model, we can estimate the optical depth knowing the pressure, temperature, and specific humidity during the time of the observations. Equation (12) shows how differential optical depth can be related to change in specific humidity via a conversion constant, $K$, which has units of inverse specific humidity because optical depth is unitless.

$$
\begin{aligned}
\Delta \tau & =\tau(t)-\tau\left(t_{0}\right)=K q(t)-K q\left(t_{0}\right) \\
& =K\left(q(t)-q\left(t_{0}\right)\right) .
\end{aligned}
$$

By substituting differential optical depth in Eq. (11) with Eq. (12) one can solve for the change in specific humidity.

$\Delta q=q(t)-q\left(t_{0}\right)=-\frac{2}{K} \ln \left(\frac{A(t)}{A\left(t_{0}\right)}\right)$.

To calculate a single conversion factor, $K$, over the test, we use the mean temperature, pressure and vapor pressure during the test, which are $290.92 \mathrm{~K}, 768.48 \mathrm{mb}$ and $12.43 \mathrm{mb}$ respectively. Using these values, we calculate the average optical depth along the observation path at $22.6 \mathrm{GHz}$ and

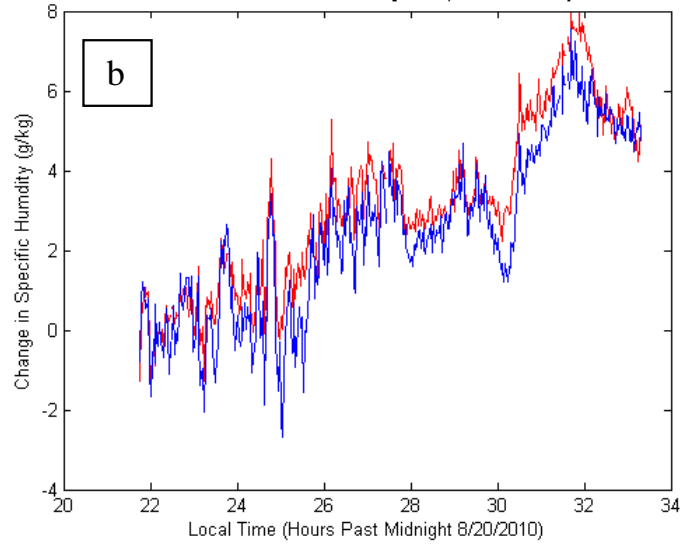

(b) Conversion to specific humidity using the MPM 93 model. The 23.5 GHz as well as the optical depths when the water vapor mixing ratio is both increased and decreased by one $\mathrm{g} \mathrm{kg}^{-1}$ relative to the mean. We then calculate the conversion factor which is the change in optical depth per change in specific humidity. The conversion factors for $22.6 \mathrm{GHz}$ and $23.5 \mathrm{GHz}$ using both models are given in Table 1. Also shown is the fractional change in the conversion factor with respect to changes in pressure and temperature. The changes in $K$ that result from the observed variations of $\pm 1.2 \mathrm{mb}$ and $\pm 2{ }^{\circ} \mathrm{C} \mathrm{re}$ spectively in pressure and temperature are $1 \%$ or less. Thus we can assume $K$ is approximately constant during the data collection with minimal error, relative to other errors.

\section{3 $22 \mathrm{GHz}$ results}

Figure 10 shows the change in specific humidity derived from the $22.6 \mathrm{GHz}$ (red) and $23.5 \mathrm{GHz}$ (blue) channels using $K$ derived from the AM 6.2 and MPM93 spectroscopic models. To generate these figures, we applied the natural logarithm to the ratio of the amplitude at each time to the amplitude averaged over the first $30 \mathrm{~min}$ of the test to determine the changes in specific humidity relative to the average 
specific humidity over the first $30 \mathrm{~min}$. A comparison of the results in Fig. 10a and $\mathrm{b}$ reveals that the derived $22.6 \mathrm{GHz}$ and $23.5 \mathrm{GHz}$ changes in specific humidity agree better with each other when the AM 6.2 model is used compared with MPM93. This suggests that the AM model may better characterize the $22 \mathrm{GHz}$ absorption line. As we improve the ATOMMS $22 \mathrm{GHz}$ system and acquire more data, we expect to be able to make definitive statements about the accuracy of microwave propagation models near the $22 \mathrm{GHz}$ absorption line.

There are fine scale differences between the $22.6 \mathrm{GHz}$ and $23.5 \mathrm{GHz}$ results. Some of this is likely caused by relative fluctuations in the transmit power between the channels. ATOMMS is equipped with transmitter power monitors that measure variations in transmit power. However, these were not calibrated well enough to be used to reduce differential fluctuations in the transmit power for this test. Some of the deviations between the two channels could also be due in part to non-uniform heating which can cause thermally sensitive components to heat up and cool off faster than others, resulting in differential variations not attributed to water vapor. In subsequent tests, we have taken steps to reduce temperature variations on the thermally sensitive components.

The comparison of all five estimates of the water vapor change from the three hygrometers and the results derived from the $22.6 \mathrm{GHz}$ and $23.5 \mathrm{GHz}$ AM 6.2 model in Fig. 11 shows overall similar behavior. The ATOMMS low band channels show a maximum overnight change in water vapor along the observation path of approximately $7 \mathrm{~g} \mathrm{~kg}^{-1}$. The two hygrometers on Mt. Bigelow show changes of $5.2 \mathrm{~g} \mathrm{~kg}^{-1}$ to $6.3 \mathrm{~g} \mathrm{~kg}^{-1}$. The differences between the ATOMMS derived water vapor and the three hygrometers appear to be dominated by spatial variations in the water vapor field itself.

In the rooftop measurements, we achieved very good agreement shown in Fig. 7 between ATOMMS and point measurement by a nearby hygrometer implying both that the ATOMMS measurements were quite accurate and that the point measurements were very similar to the average water vapor along the $820 \mathrm{~m}$ path. Thus the point measurement provided excellent "ground truth" against which we could compare and assess ATOMMS. In contrast, the disagreement between the three measurements of water vapor in Fig. 9 clearly indicate that the "ground truth" knowledge of water vapor along the $5.4 \mathrm{~km}$ path between the two ATOMMS instruments above the valley at the top of the Catalina mountains is much harder to establish than it was for the $820 \mathrm{~m}$ path length on campus. One reason is that the longer path allows for more variation along the path. Another is the steep topography which can result in much stronger diurnal variations, such as the stratification and substantial variation with height as evidenced in the three hygrometer measurements during this test. Moisture exhibits large temporal and spatial variations, causing comparisons of point and path average measurements to disagree. Much of the differences in the smaller scale variations can be attributed to the fact

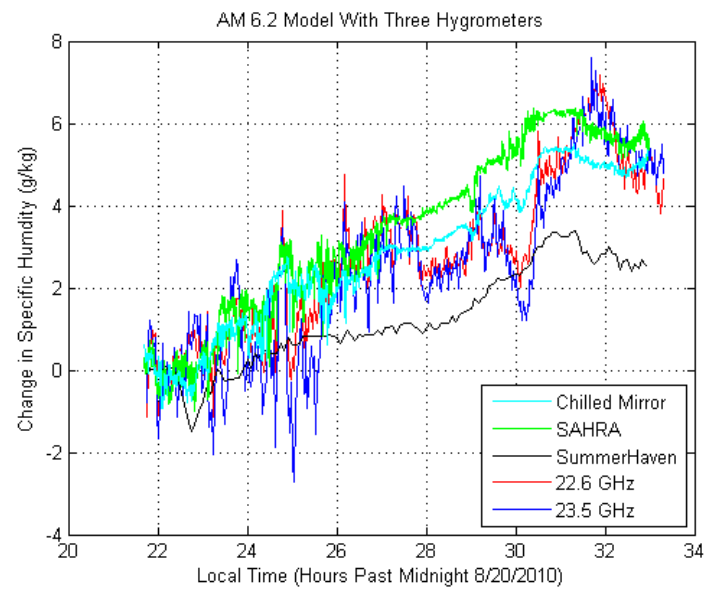

Fig. 11. Five curves each representing a particular measured change in specific humidity. The ATOMMS $22.6 \mathrm{GHz}$ and $23.5 \mathrm{GHz}$ channel amplitudes have been converted to specific humidity using the AM 6.2 spectroscopic model.

that the three hygrometers are point measurements whereas ATOMMS observes variations of water vapor along a path.

\section{Mountaintop measurements during a storm on 28 August 2010}

On 28 August 2010 we made a second set of measurements between Mt. Lemmon and Mt. Bigelow. During this test, a storm passed through that allowed us to measure the opacity of liquid cloud and rain droplets with ATOMMS. Figure 12 (left hand side) shows the temperature and dew point temperature measured by our chilled mirror hygrometer on Mt. Bigelow. We began recording ATOMMS data at noon. Water vapor varied little over the next $2.5 \mathrm{~h}$ until the onset of the storm. Rapid cooling (likely associated at least in part with evaporative cooling of rain) began around 14:25 h and was followed by a sharp increase in dew point around $14: 45 \mathrm{~h}$.

Figure 13 shows the change in opacity measured by ATOMMS at $197 \mathrm{GHz}$ during the storm. These variations are due to a combination of changes in water vapor, cloud liquid water and rain. Variations associated with changes in temperature and pressure are very minor. In order to understand the cause of the changes in optical depth at $197 \mathrm{GHz}$ we also examined radar and optical depths at visible wavelengths.

Because radar backscatter measurements are sensitive to the largest droplets whereas the $197 \mathrm{GHz}$ measurements are sensitive to droplets of all sizes, we proceed as follows: (1) derive a relationship between radar reflectivity and the two free variables describing an exponential particle size distribution for a particular reflectivity vs. rain rate relation; (2) use that relation to estimate the $197 \mathrm{GHz}$ opacity due to rain for the measured reflectivity vs. time; (3) subtract the 

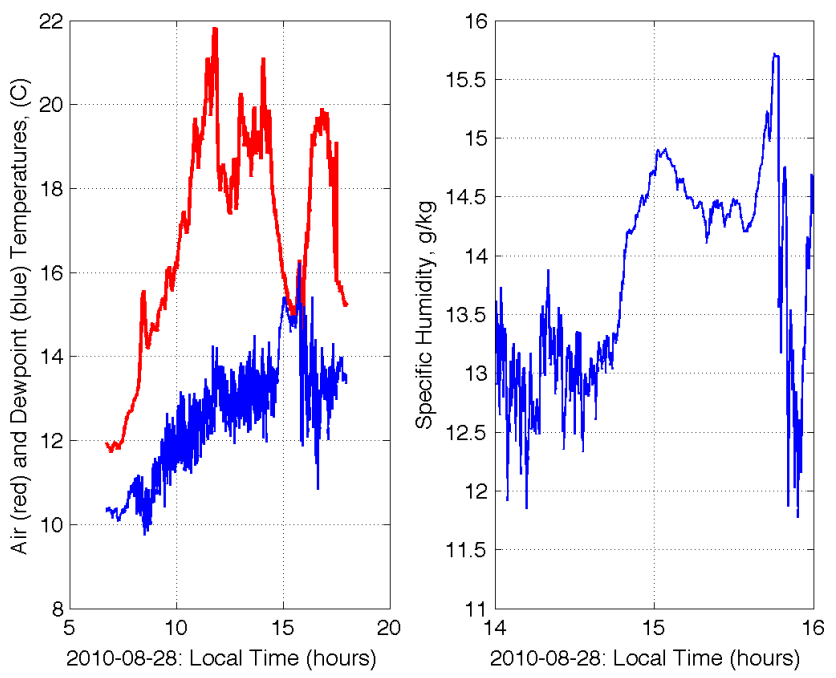

Fig. 12. Left panel: measurements of the temperature and dew point from the chilled mirror hygrometer on Mt. Bigelow. Right panel: specific humidity from 14:00 to 16:00 LT.

estimated rain opacity from the total opacity measured by ATOMMS to determine the $197 \mathrm{GHz}$ opacity due to clouds and (4) convert that opacity to a cloud liquid water content (LWC). Visible wavelength opacities derived from photographs taken during the storm also provide useful independent constraints on the particle sizes.

\subsection{RADAR data}

The WSR-88 radar in southeastern Arizona observed the storm's evolution with $5 \mathrm{~min}$ resolution, $250 \mathrm{~m}$ range resolution and $0.5^{\circ}$ azimuthal resolution at a distance of about $60 \mathrm{~km}$ from the ATOMMS instruments. A radar measures signals backscattered from liquid droplets much smaller than the wavelength and hence in the Rayleigh regime. The total backscatter is the sum over all backscattering particles. Treating the particle sizes as a continuous distribution yields the reflectivity, $Z$, which is expressed as the following integral over the particle size distribution (e.g. Fraile and Fernandez-Raga, 2009)

$Z=\int n(D) D^{6} \mathrm{~d} D$

where $D$ is the particle diameter in $\mathrm{mm}$, and $n(D)$ is the droplet size distribution in units of $\mathrm{mm}^{-1} \mathrm{~m}^{-3}$. The reflectivity is typically given in $\mathrm{dBz}$ which is $10 \log _{10}\left(Z / Z_{0}\right)$ where $Z_{0}=1 \mathrm{~mm}^{6} \mathrm{~m}^{-3}$. Figure 14 shows the time evolution of the radar backscatter along the line of sight between our ATOMMS instruments on Mt. Lemmon and Mt. Bigelow separated into 65 pixels along the path. Two elevation scans from the radar are shown at $0.88^{\circ}$ and $1.28^{\circ}$. While the $0.88^{\circ}$ elevation scan is just above Mt. Lemmon and therefore closer to the actual volume sampled by ATOMMS, it clearly shows

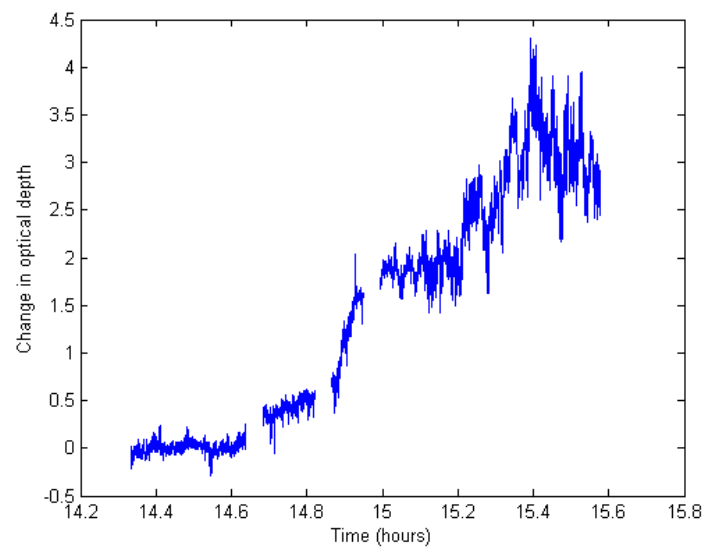

Fig. 13. Plot of change in optical depth versus time measured at $197 \mathrm{GHz}$ given as $-2 \times \ln \left(A_{\text {norm }}(t)\right)$ where the amplitude is normalized to the amplitude just before the storm hit. Outages were caused by software problems that necessitated manual restarts.

more ground clutter than the $1.28^{\circ}$ elevation scan, which is a bit higher above the surface. Thus the $1.28^{\circ}$ scan is less noisy.

\subsection{ATOMMS opacity due to liquid water}

The change in opacity measured by ATOMMS at $197 \mathrm{GHz}$ shown in Fig. 13 is due primarily to condensed liquid water because the water vapor is very close to saturation at the beginning of the storm and does not change much during the storm. The increase in opacity is therefore due to a combination of rain drops and cloud droplets. The cloud droplets are spherical. The rainfall rate measured during the course of this storm by two different gauges on the mountain, one at Summerhaven and one at the SAHRA flux tower was approximately $0.5 \mathrm{~mm} \mathrm{~h}^{-1}$. This is comparable to the radar-derived estimates (Fig. 15), which is a light rain. Therefore we assume the rain droplets are mostly spherical which allows us to use Mie theory to understand the electromagnetic crosssections of the droplets as a function of wavelength.

The extinction coefficient, $k$, due to spherical particles is given below (e.g. Bohren and Huffman, 1983)

$k(D, \lambda)=\int \sigma(D) n(D) \mathrm{d} D=\int Q(D, \lambda) \frac{\pi}{4} D^{2} n(D) \mathrm{d} D$

where $Q(D, \lambda)$ is the Mie extinction efficiency and $\lambda$ is the signal wavelength. The extinction efficiency is the sum of the scattering and absorption efficiencies, $Q_{\text {ext }}=Q_{\text {abs }}+Q_{\text {scat }}$. The Mie extinction efficiency at the ATOMMS frequency of $197 \mathrm{GHz}$ for cloud and rain particles is shown in Fig. 16.

The attenuation of the $197 \mathrm{GHz}$ observations and the radar backscatter depend on the particle size distribution. Researchers have described the size distribution of raindrops by a number of analytic expressions including the exponential, gamma and log-normal distributions. Williams and Gage (2009) examined seven distributions consisting of two 

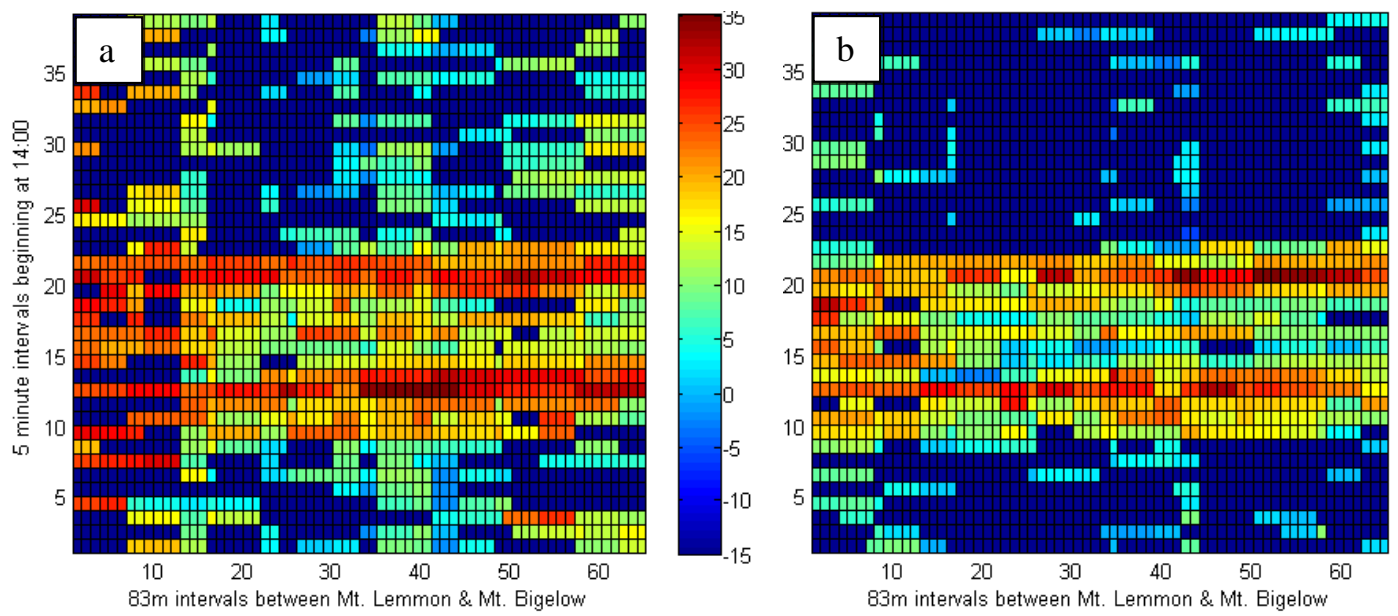

Fig. 14. Radar measured reflection in $\mathrm{dBz}$, at radar elevation of (a) $0.88^{\circ}$ and (b) $1.28^{\circ}$, time is vertical scale in units of 5 min intervals beginning at 14:00. The left edge is Mt. Lemmon, and right hand edge is Mt. Bigelow. The $5.4 \mathrm{~km}$ path between them is divided into 65 equal intervals. The units of the color scale shown in the center are dBz.

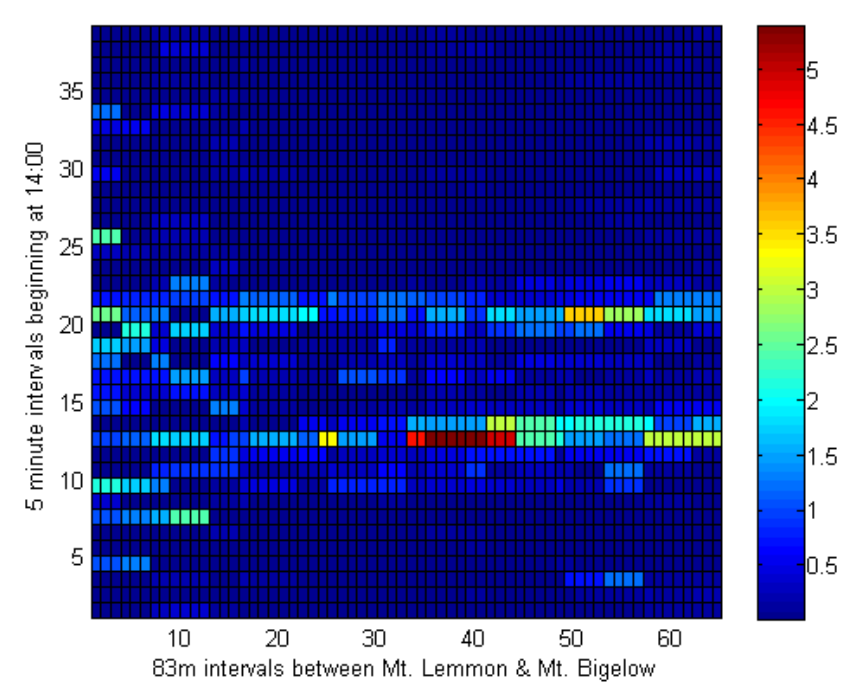

Fig. 15. Rainrate in $m \mathrm{~m} \mathrm{~h}^{-1}$ derived from the Tucson WSR-88 radar data via the standard $Z-R$ relation. The $\mathrm{x}$ - and $\mathrm{y}$-axes are the same as in Fig. (14). The units of the color scale are $\mathrm{mm} \mathrm{h}^{-1}$.

versions of exponential including the original distribution of Marshall and Palmer (1948), four versions of the gamma distribution and one log-normal distribution. They concluded that other than the original Marshall Palmer distribution which was systematically in error for the tropical conditions they were considering, the other six distribution were essentially equivalent in terms of accuracy. Therefore we choose to use an exponential size distribution for raindrops as defined in Eq. (16) to provide a simple, realistic size distribution with only two degrees of freedom, $n_{0}\left(\mathrm{~m}^{-3}\right)$, the number density of droplets and $D_{0}(\mathrm{~m})$, the mean droplet diameter.

$n(D)=\frac{n_{0}}{D_{0}} e^{-\frac{D}{D_{0}}}$
Using an exponential distribution, Eq. (14) can be rewritten as

$$
Z=\int n(D) D^{6} \mathrm{~d} D=\frac{n_{0}}{D_{0}} \int e^{-\frac{D}{D_{0}}} D^{6} \mathrm{~d} D .
$$

We use an upper limit of $6 \mathrm{~mm}$ in diameter based on laboratory measurements. Equation (17) does not include the flattening effect for the larger droplets. While the present ATOMMS instrument transmits and receives circularly polarized radiation, modifying the $197 \mathrm{GHz}$ receive system to measure both linear polarizations would measure the effects of the flattening.

\subsection{Estimating rain mass and opacity from the weather radar}

The radar provides a time sequence of one variable, $Z$. To obtain a time sequence of the two variables, $n_{0}$ and $D_{0}$, in the exponential distribution from the radar data, we need at least one more constraint. As that constraint, we the radarrain rate relation, $Z=a R^{b}$. The obvious $a$ and $b$ constants are the US National Weather Service's standard conversion between the reflected power measured by a WSR-88D radar and rain rate

$Z=300 R^{1.4}$

where $Z$ is in $\mathrm{mm}^{6} \mathrm{~m}^{-3}$ and $R$ is in $\mathrm{mm} \mathrm{h}^{-1}$ (Fulton et al., 1998). Researchers have found some variation in these constants with conditions. Morin et al. (2005) used $Z=655 R^{1.4}$ in the Walnut Gulch region in Southeast Arizona, arguing that large evaporation of the droplets was responsible for much larger value of $a$. Because our ATOMMS measurements were taken approximately $1500 \mathrm{~m}$ higher than the rain gauge measurements in Walnut Gulch and close to cloud 


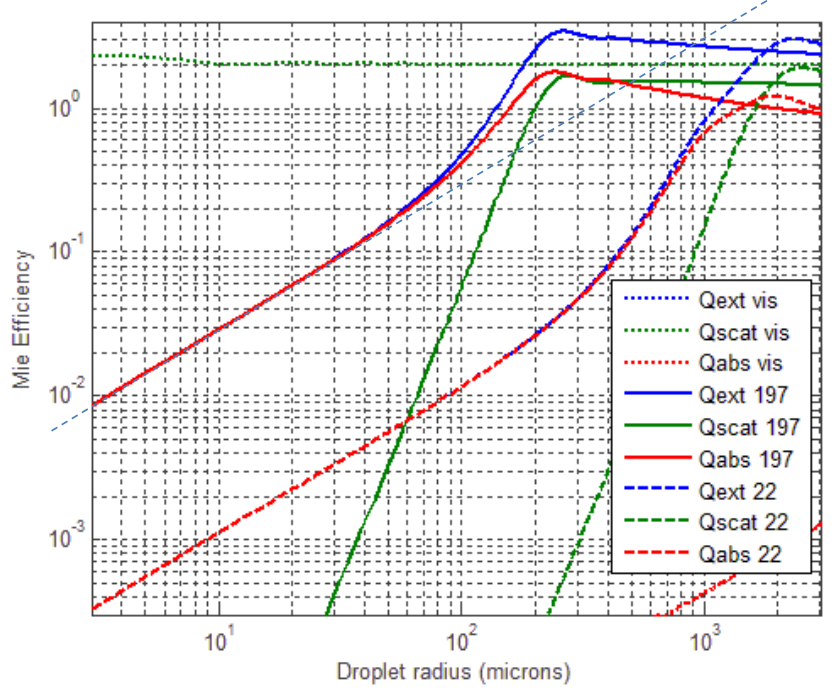

Fig. 16. Mie scattering efficiency at $22 \mathrm{GHz}, 197 \mathrm{GHz}$ and visible wavelengths versus the water droplet radius in microns. The index of refraction of the water used here is $5.5+2.9 \mathrm{i}, 2.83+1.24 \mathrm{i}$ and $1.3+1 \mathrm{e}-8 \mathrm{i}$ at $22 \mathrm{GHz}, 197 \mathrm{GHz}$ and visible light respectively. The thin blue dashed line shows Rayleigh behavior at $197 \mathrm{GHz}$ extrapolated to larger radii. At $r=0.25 \mathrm{~mm}$, the $197 \mathrm{GHz}$ extinction due to Mie scattering and absorption is approximately 5 times larger than the extrapolated Rayleigh regime behavior. A typical cloud particle has a radius of 5 microns.

base such that evaporation was much smaller than the conditions examined by Morin et al., we use the standard relation (18). We have also examined different constants. Ochou et al. (2011) found the variations in the $Z=a R^{b}$ relation were primarily in the constant, $a$, finding 1-sigma variations in $a$ of $30 \%$ and $b$ of $7 \%$. We therefore also included $\pm 30 \%$ variations in $a$, specifically with $a=231$ and 390 .

To determine how $n_{0}$ and $D_{0}$ vary with $Z$ for a given $Z=a R^{b}$ relation, we calculate the reflectivity from Eq. (17) and the rain rate from Eq. (18) as a function of $n_{0}$ and $D_{0}$ according to

$R=\frac{6 \pi}{10000} \int_{0}^{D_{\max }} N(D) D^{3} v(D) \mathrm{d} D$

where $v$ is the droplet terminal fall speed determined from laboratory measurements and approximated as

$v(D)=\left(9.65-10.3 e^{-0.6 D}\right)\left(\frac{\rho}{\rho_{0}}\right)^{-0.4}$

where $\rho$ is the air density and $\rho_{0}$ is the air density at sea level (Gunn and Kinzer, 1949; Atlas et al., 1973). From these we determine the relation between $n_{0}$ and $D_{0}$ that results in $Z=a R^{1.4}$ for $a=231,300$, and 390 .

Given $n_{0}$ and $D_{0}$ we combine the exponential distribution with the Mie scattering extinction efficiency, $Q$, shown in

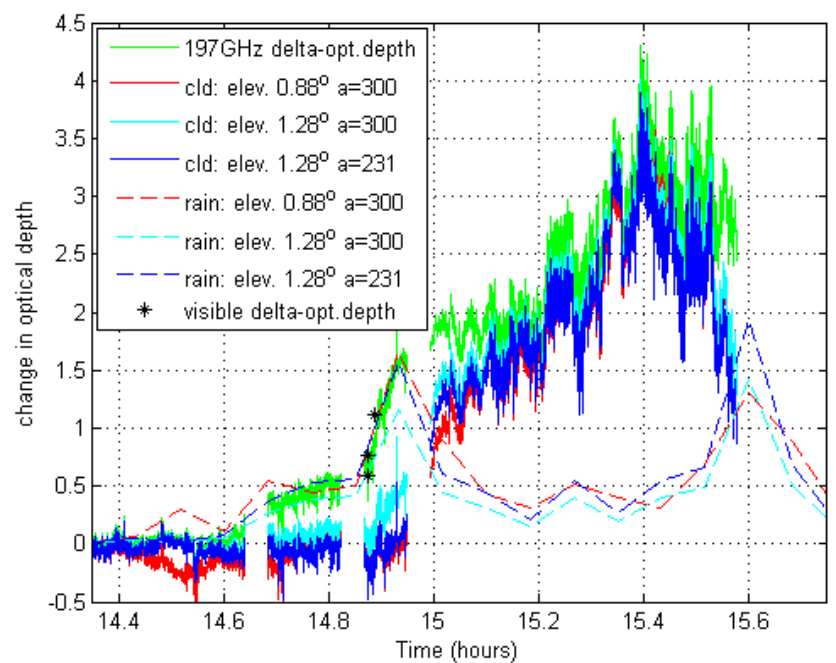

Fig. 17. Plots of the change in optical depth during the storm. Green is the change in optical depth measured at $197 \mathrm{GHz}$. The optical depths due to rain estimated from the WSR-88 radar are shown as dashed lines. The estimated optical depths due to clouds are shown as solid red, cyan and blue lines, which are calculated as the difference between the $197 \mathrm{GHz}$ measurements and that due to rain. The asterisks are changes in optical depth at visible wavelengths derived from photographic images taken during the storm. See text for details.

Fig. 16 to calculate the expected extinction coefficient and, from that, the expected extinction coefficient of the rain at the ATOMMS wavelength.

$k_{\text {rain }}=\int \sigma(r) n_{\text {rain }}(r) \mathrm{d} r=\int Q(r, \lambda) \pi r^{2} \frac{n_{0}}{D_{0}} e^{-\frac{2 r}{D_{0}}} \mathrm{~d} r$

where $r$ is the raindrop radius and $n_{\text {rain }}$ is the dropsize distrribution of the raindrops. In applying Eq. (21) to estimate the loss of power from the radiated signal, we make the simplifying assumption of ignoring any energy forward scattered back into the beam.

As noted, we considered three different values of the constant, $a$, in the $Z=a R^{1.4}$ equation. Of the six possible combinations of the three $a$ values and the two elevation angles, $0.88^{\circ}$ and $1.28^{\circ}$, Fig. 17 shows the three combinations that provide the most reasonable results and provide some indication of the uncertainty. Based on the nominal $Z(R)$ relation of Fulton et al. (1998) the $a=655$ result Morin et al. (2005) found under conditions of large evaporation and the close proximity of the ATOMMS beam to cloud base, we anticipate a best value of $a=300$ or perhaps slightly less. The similarity of the measured $197 \mathrm{GHz}$ and visible wavelength optical depths at the onset indicates the rapid increase in opacity is due to rain such that the radar-based optical depths at $197 \mathrm{GHz}$ should be close to the measured $197 \mathrm{GHz}$ values. However, when optical depths at the storm onset are estimated using $a=390$, they were too small at both the $0.88^{\circ}$ and $1.28^{\circ}$ elevation angles indicating the $a=390$ value is too 
large. The results for $a=300$ at $0.88^{\circ}$ elevation angle and $a=231$ at $1.28^{\circ}$ elevation angle were very similar to one another and to the $197 \mathrm{GHz}$ measurements. The $a=300,1.28^{\circ}$ elevation opacities were a bit smaller but still credible. The peak rain opacity during the onset for $a=231$ and an elevation of $0.88^{\circ}$ was 2.2 , which is $50 \%$ larger than the measured $197 \mathrm{GHz}$ opacity and therefore too large. We suspect the best value of $a$ is somewhat smaller than 300 but not as small as 231. These results further suggest that a systematic evaluation of the $Z(R)$ relation could be accomplished using a combination of radar, ATOMMS, visible wavelength measurements and disdrometers.

The opacity measured by ATOMMS due to liquid water is the sum of the opacity due to rain and cloud

$$
\begin{aligned}
\tau_{197} & =\int k_{197} \mathrm{~d} z=\int\left(k_{197 \text { rain }}+k_{197 \text { cloud }}\right) \mathrm{d} z \\
& =\tau_{197 \text { rain }}+\tau_{197 \text { cloud }} .
\end{aligned}
$$

Therefore the opacity due to cloud can be estimated as

$\tau_{197 \text { cloud }}=\tau_{197}-\tau_{197 \text { rain }}$

where $\tau_{197}$ is the optical depth measured directly by ATOMMS and $\tau_{197 \text { rain }}$ is the optical depth due to rain which is estimated from the radar data as described above. Figure 17 shows the estimated cloud optical depth versus time, derived by subtracting the rainfall opacity estimated via Eq. (21) from the measured optical depth at $197 \mathrm{GHz}$.

\subsection{Derivation of cloud liquid water content}

The cloud particles are sufficiently small that the attenuation at $197 \mathrm{GHz}$ is due to absorption in the Rayleigh regime such that $Q$ is proportional to $x=2 \pi / \lambda$ which is known as the size parameter. We can therefore simplify the $197 \mathrm{GHz}$ extinction coefficient due to clouds, $k_{\text {cloud }}$, to be

$$
\begin{array}{r}
k_{\text {cloud }}=\int_{\text {cloud }} \sigma(r) n(r) \mathrm{d} r=\int 0.7 x \pi r^{2} n(r) \mathrm{d} r \\
=0.7 \frac{6 \pi}{4 \lambda p} \int \frac{4}{3} \pi r^{3} n(r) p \mathrm{~d} r=1.05 \frac{\pi}{\alpha p} \mathrm{LWC}
\end{array}
$$

where LWC is cloud liquid water content and $\rho$ is the density of liquid water. The cloud optical depth, $\tau_{\text {cloud }}$, measured by ATOMMS along the path between the two mountains is defined as

$\tau_{\text {cloud }}=\int k_{\text {cloud }} \mathrm{d} z=\bar{k}_{\text {cloud }} L=1.05 \frac{\pi}{\alpha p} \overline{\mathrm{LWC}}_{\text {cloud }} L$

where the overbars indicate the average along the path, $L$, between the two ATOMMS instruments. Thus, from $\tau_{\text {cloud, }}$, we can estimate the average cloud LWC along the path as

$\overline{\mathrm{LWC}}_{\text {cloud }}=\frac{\tau_{\text {cloud }} \alpha p}{1.05 \pi L}$.

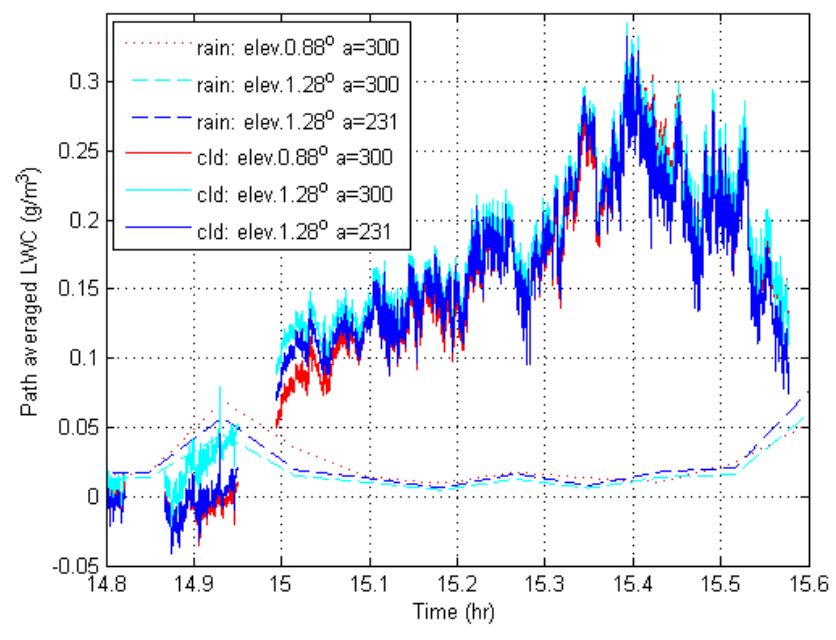

Fig. 18. Estimated average liquid water content in the rain and clouds along the $5.4 \mathrm{~km}$ path between the mountains.

Given that typical values of continental cloud LWC are $0.3 \mathrm{~g} \mathrm{~m}^{-3}$, these estimated cloud LWC shown in Fig. 18 are quite reasonable. The peak near 15:24h may be associated with the cloud extending along the entire path between the two ATOMMS instruments.

Utilizing the combined radar and $197 \mathrm{GHz}$ datasets is limited by several factors. First, the radar only updates every 5 min. So the radar data in Figs. 17 and 18 is only every $5 \mathrm{~min}$ and linearly interpolated between the points every $5 \mathrm{~min}$. Second, the radar sees a larger volume than the ATOMMS beam's line of sight between the two mountains. In particular, it sees the region above the ATOMMS beam. At the onset of the rain, the radar observes rain falling above the $197 \mathrm{GHz}$ beam that then falls into the ATOMMS beam causing the rapid increase in opacity in the $197 \mathrm{GHz}$ data around 14:52. This difference in sampling volume can cause the apparent opacity due to rain to be larger than what was measured by ATOMMS. Finally, the radar is also degraded by surface reflections (known as ground clutter) that make the radar data noisy as is visible in Fig. 14.

\subsection{Visual optical depth derived from photographs}

Photographs taken from Mt. Bigelow looking toward Mt. Lemmon during the storm provide additional constraints about scattering caused by the rain and clouds at visible wavelengths that have proven useful, particularly at the onset of the storm as shown in Fig. 19. As discussed in Appendix A, in each photograph, four points were used, calibration location, near location, medium location and a far location as marked in Fig. 19. The distances to the calibration, near, medium and far location are $0.010 \mathrm{~km}, 0.30 \mathrm{~km}$, $0.78 \mathrm{~km}$ and $5.4 \mathrm{~km}$ respectively. In each picture, the mean red, green and blue intensities were calculated at each of the four locations. 

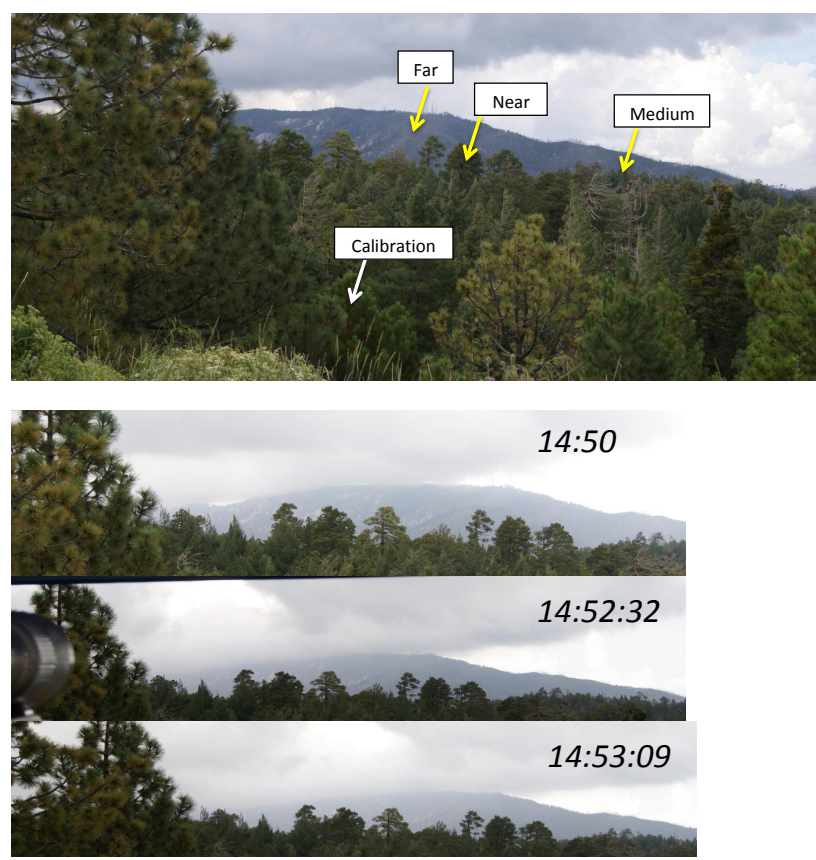

Fig. 19. View of Mt. Lemmon from Mt. Bigelow at visible wavelengths showing the evolving opacity at the onset of the storm.

At visible wavelengths, absorption by liquid water is very small and the effect of water droplets on the light is due almost entirely to scattering. Since the sky at the time was covered with clouds, the light source, $S$, along the path was scattered and can be approximated as homogeneous, that is, equal in all directions along the path. Under these conditions, the intensity of light measured in the photographs is given in Eq. (25) (see Appendix A).

$I=I_{0} e^{-\tau_{\max }}+S\left(1-e^{-\tau_{\max }}\right)$.

This relationship shows that the received intensity is the sum of two terms. The first term is the intensity of the light that has reflected off the object being imaged, $I_{0}$, that is then attenuated by scattering of light out of the beam path as the light propagates to the camera. The second term is the increase in intensity of light in a given pixel due to scattering into the beam path. The intensity of pure scattered light, $S$, is taken to be the maximum value of the pixels in a given image. Equation (27) shows that as the opacity increases, the light from the object being imaged is scattered out of the beam and replaced by light scattered into the beam from the scattered sunlight.

The simultaneous opacity measurements at $\sim 0.5$ micron and $1.5 \mathrm{~mm}$ wavelengths provide strong constraints on the particle sizes along the path during the onset of the storm and in particular indicate whether the drops are rain or cloud droplets. The extinction coefficient at each wavelength is given by Eq. (15). Since the signals at both wavelength bands propagate through the same atmosphere and particles, the only difference in the measured opacities is the difference in the Mie scattering efficiencies, the $Q_{197}$ and $Q_{\text {vis }}$ terms. As shown in Fig. 16, $Q_{197}$ ranges from 0.015 for 5 micron radius cloud droplets to 3.5 for $0.25 \mathrm{~mm}$ radius rain droplets whereas $Q_{\text {vis }}$ is approximately 2 for both cloud and rain droplet sizes. Figure 17 shows that the measured changes in opacity at $197 \mathrm{GHz}$ and visible wavelengths between 14:50 h and 14:53 $\mathrm{h}$ were quite similar, exhibiting simultaneous rapid increases. The similar changes in optical depth indicate that $Q_{\text {vis }}$ and $Q_{197}$ are comparable which can only be true if the water droplets along the path at the onset of storm were primarily rain and not cloud droplets. The agreement in the timing and magnitude also provides a strong validation of the ATOMMS opacity measurements.

\subsection{Interpretation of what happened during the storm}

\subsubsection{Change in optical depths just prior to storm $(14: 35 \mathrm{~h}$ to $14: 50 \mathrm{~h})$}

From $14.6 \mathrm{~h}$ to $14.8 \mathrm{~h}$, the optical depth at $197 \mathrm{GHz}$ increased from 0 to 0.5 . During this time interval, our chilled mirror hygrometer on Mt. Bigelow measured an increase in water vapor from $15.6 \mathrm{hPa}$ to $17.5 \mathrm{hPa}$ as it rose to saturation (Fig. 12). If this same increase occurred over the entire path, the corresponding increase in optical depth at $197 \mathrm{GHz}$ would have been 0.53 which is about the increase measured by ATOMMS over this period. This may not be the entire explanation because the WSR-88D RADAR also observed a small increase in reflectivity in this time interval at both the $0.88^{\circ}$ and $1.28^{\circ}$ elevation scans (Fig. 14) indicating ATOMMS should have measured some increase in opacity associated with light rain. The RADAR data is noisy particularly at the $0.88^{\circ}$ elevation scan which sees a larger reflectivity and ground clutter.

So the measured increase in $197 \mathrm{GHz}$ opacity over this period is likely due to an increase in the water vapor concentration and may include some light rainfall. The two may in fact be related because any evaporation of rainfall would increase the vapor concentration. Had the tuning of the ATOMMS instrument operated correctly during this period, we could have separated the vapor and condensed water effects from the measurements themselves. Hopefully we will have another opportunity to do so.

\subsubsection{Rapid increase in attenuation associated with the first rain $(14: 50 \mathrm{~h}$ to $15: 00 \mathrm{~h})$}

The rapid increase of approximately 1.2 in $197 \mathrm{GHz}$ optical depth over the $14: 50 \mathrm{~h}$ to $15: 00 \mathrm{~h}$ interval is too large to be associated with water vapor given that the air is already very close to saturation. The opacity increase must therefore be due to condensed moisture in the form of clouds and rain. The storm's onset was probed at three very different wavelengths by ATOMMS, the WSR-88D radar in 
southeastern Arizona and in photographs. The very similar magnitude and rapid rise in the $1.5 \mathrm{~mm}$ and visible wavelength opacities indicates the opacity increase at the onset of the storm was caused by rain rather than via advection of clouds across the path, consistent with the simultaneous radar rain measurements.

This rapid onset period therefore gives us the additional constraint that the radar-derived rain opacity at $197 \mathrm{GHz}$ must equal the measured $197 \mathrm{GHz}$ opacity at the storm onset. We varied the constant, $a$, in the $Z(R)$ relation by $\pm 30 \%$. In converting the radar measurements at the $0.88^{\circ}$ elevation angle to $197 \mathrm{GHz}$ opacity, the nominal value of $a=300$ yields a rapid rise in opacity very similar to the measured $183 \mathrm{GHz}$ opacity. The conversion of radar measurements at $1.28^{\circ}$ to $197 \mathrm{GHz}$ opacity is closest to the measured $197 \mathrm{GHz}$ opacity using $a=231$. The $0.88^{\circ}$ radar measurements could be too high because of ground clutter. The $1.28^{\circ}$ measurements could be an underestimate because the measurements are a few hundred meters above the ATOMMS signal path. Because the ATOMMS measurements are so close to cloud base at the storm onset, therefore with very little evaporation, one could imagine that the best estimate of $a$ is somewhat smaller than the NWS nominal value of 300 . This suggests that visible/near-IR measurements simultaneous with ATOMMS and weather radar measurements can be used to assess and refine the $Z(R)$ relation under different conditions. Combining these with a drop distrometer would provide further information on the drop size distribution and its evolution as the drops fall from the cloud to the ground.

Furthermore, a fully functioning ATOMMS system would provide sufficient spectral information to simultaneously measure the variations in water vapor and condensed water and perhaps temperature (via lineshape) to measure conversion between the phases during the rain/virga. This would have been useful here because as rain fell into the subsaturated clear air below cloud base, some evaporated, cooling the air and lowering the saturation vapor pressure of the air which could then cause some of the evaporated moisture to recondense into cloud droplets. This is qualitatively consistent with the rapid decrease in temperature and increase in dew point measured by the hygrometer as the storm approached.

\subsubsection{Cloud with little precipitation $(15: 00 \mathrm{~h}$ to $\sim 15: 12 \mathrm{~h}$ )}

From $15: 00 \mathrm{~h}$ to $15: 12 \mathrm{~h}$, the $197 \mathrm{GHz}$ opacity remained nearly constant, approximately 1.8 higher than before the storm onset while the radar reflectivity was small. The portion of the photographs where Mt. Lemmon had been visible became white-gray indicating visible scattering opacities greater than 2. Together, these indicate clouds are present along the path together with a little rain. Over this period, the average cloud LWC along the path increases from about $0.10 \mathrm{~g} \mathrm{~m}^{-3}$ to $0.15 \mathrm{~g} \mathrm{~m}^{-3}$.

\subsubsection{Cloud + precipitation $(\sim 15: 12 \mathrm{~h}$ to $\sim 15: 40 \mathrm{~h})$}

By 15:20 h, Mt. Bigelow was in the clouds, experiencing light rain. The peak optical depth (relative to the pre-storm period) of 4 occurred near 15:24 h. As Fig. 19 shows, the path averaged liquid water content of the cloud at this time reached about $0.3 \mathrm{~g} \mathrm{~m}^{-3}$. According to our field notes, the cloud began to clear from the Mt. Lemmon site at 15:30 h, while Mt. Bigelow remained in cloud until about 15:45 h. Given the storm's approximate direction of motion shown in Fig. 8, the decrease in cloud LWC after 15:30 h was likely associated, at least in part, with a decrease in the extent of the cloud along the signal path as the end of the storm passed through.

\section{Summary and discussion}

In summary, we have presented the first ground based test results from the ATOMMS prototype instrument. The rooftop spectra near the $183 \mathrm{GHz}$ line showed that ATOMMS is a sensitive spectrometer capable of distinguishing between and evaluating two standard spectroscopic models, clearly showing the superiority of the AM 6.2 model over the MPM93 model. The results demonstrated ATOMMS' sensitivity to lineshape that will be a key to making orbiting ATOMMS observations independent of climate models, unlike GPS RO. These measurements indicate the standard deviation of the amplitude errors are $\sim 0.3 \%$ which is quite promising.

We showed that in these surface observations ATOMMS can measure changes in water vapor quite accurately. Our $183 \mathrm{GHz}$ rooftop measurements demonstrated ATOMMS ability to measure changes in water vapor to about $0.05 \mathrm{mb}$ (Fig. 7). We showed the first $22 \mathrm{GHz}$ mountaintop results demonstrating the ability to probe that line to determine changes in water vapor over a $5.4 \mathrm{~km}$ path. Measuring changes in water vapor relative to the minimum water vapor is the same approach that will be used with the aircraft and satellite occultations. In these cases, the minimum water vapor along the path relative to which changes will be measured is a few parts per million for high altitude aircraft to aircraft occultations or zero in the satellite to satellite occultations. So, in the satellite case, the changes in water vapor determined by ATOMMS will equal the absolute water vapor.

We also presented mountaintop measurements made during a storm in late August 2010. These measurements confirm that ATOMMS signals penetrate through the rain and clouds as expected. By combining ATOMMS measurements with WSR-88D data and photographs together with Mie theory, we were able to make sense of the mountaintop storm measurements and verify that the ATOMMS measurements were reasonable. Optical depths derived from photographs combined with ATOMMS measurements revealed that the storm began with rainfall rather than clouds along the 
ATOMMS signal path. The whiting out of the photographs caused by scattering by rain and cloud droplets reminds us of the limitations of remote sensing at visible wavelengths on a planet with $60 \%$ to $70 \%$ global cloud cover (Rossow and Shiffer, 1999). Remote sensing at IR wavelengths is quite powerful for both weather and climate monitoring and forecasting and in fact critical for monitoring outgoing longwave radiation (OLR) to space. However, its limited ability to penetrate clouds leads to preferential sampling of drier, cloudfree air that creates dry biases for instance in upper tropospheric humidity (UTH) (Lazante and Gahrs, 2000). John et al. (2011) note that The clear-sky HIRS (High Resolution Infrared Radiation Sounder) measurements are sampling meteorologically unusual situations of cloud free conditions, so they only represent a limited aspect of the climate system. Interpreting trends in such biased observations is inherently difficult and ambiguous because changes in the sampling of clouds, whose properties are likely to change in a changing climate, will cause shifts in the sampling biases over time (e.g. John et al., 2011). Unambiguously monitoring and understanding trends in the climate state can only be accomplished by sampling the entire range of behavior of the Earth's atmosphere, which can only be achieved at wavelengths long enough to routinely penetrate through clouds.

The ATOMMS measurements will work well to optical depths of $\sim 10$. Therefore the high band signals will penetrate through and measure the properties of clouds similar to those encountered here for path lengths up to about $14 \mathrm{~km}$ but not over much larger distances. The $22 \mathrm{GHz}$ signals will propagate through much longer paths through clouds because the extinction coefficient due to liquid water is about 30 times smaller at $20 \mathrm{GHz}$ than $200 \mathrm{GHz}$ (Fig. 16). In fact, the liquid water opacity at $22 \mathrm{GHz}$ over the $5.4 \mathrm{~km}$ path of this test was too small to measure reliably until we have working transmitter power monitors.

As discussed by Kursinski et al. (2009), with sampling across the line, ATOMMS measurements will provide the spectral information needed to separate the vapor from the liquid water changes. Unfortunately, during this mountaintop test, the $183 \mathrm{GHz}$ band tuning measurements did not work well and we could not yet demonstrate this capability. In future tests, we will demonstrate this ability to separate the vapor from the liquid and better constrain the physical processes at work. For instance, it will characterize rain evaporation below cloud base via the resulting increase in water vapor content and decrease in temperature (measured via changes in linewidth), and potential subsequent condensation into clouds thereby lowering cloud base and vertically transferring energy in the atmosphere.

Another signature of rain that we will look for when the full ATOMMS instrument is running is a relative phase shift between the shorter and longer wavelength signals associated with scattering by raindrops at the shorter wavelengths (Fig. 16). The ability to do so would provide additional constraints on the raindrop size distribution. Based on the new constraints and insight gained from these tests, we are now developing a weatherproof enclosure that will allow us to leave the ATOMMS instruments operating on the mountain for longer periods to observe weather variations and storms.

Overall, these results demonstrate some of the capabilities of ATOMMS as an open air, differential microwave spectrometer that accurately determines changes in vapor pressure, temperature and pressure and liquid water along the path between the ATOMMS transmitter and receiver.

\section{Appendix A}

\section{Derivation of optical depths at visible wavelengths}

At visible wavelengths, absorption by liquid water is very small and the effect of water droplets on the light is due almost entirely to scattering. Equation (A1) shows that the received intensity is the sum of two terms. The left hand term is the intensity of the light that has reflected off the object being imaged that is then attenuated by scattering of light out of the beam path as the light propagates to the camera. The right hand term is increase in intensity of light in that pixel due to scattering into the beam path.

$I=I_{0} e^{-\tau_{\max }}+\int_{\tau^{\prime}=0}^{\tau^{\prime}=\tau_{\max }} S e^{\int_{\tau^{\prime \prime}=\tau^{\prime}}^{\tau^{\prime \prime}=\tau \max }}-\mathrm{d} \tau^{\prime \prime} \mathrm{d} \tau^{\prime}$

where $I_{0}$ represents the intensity of the object without attenuation or brightening and $S$ is intensity of pure scattered light and is taken to be the maximum value of the pixels in a given image. Assuming the scattered light source, $S$, is homogeneous, that is, equal in all directions along the path yields the relationship given in Eq. (A2).

$I=I_{0} e^{\tau \max }+S\left(1-e^{-\tau_{\max }}\right)$.

Equation (A2) shows that as the opacity increases, the light from the object being imaged is scattered out of the beam and replaced by light scattered into the beam from the cloud. Equation (A2) can be written as

$(I-S)=\left(I_{0}-S\right) e^{-\tau_{\max }}$.

Taking the ratio of Eq. (A3) for the far location from two different images yields the change in optical depth shown in Eq. (A4). This assumes that $\left(I_{0}-S\right)$ does not change with time.

$$
\begin{aligned}
& \ln \left(\frac{(I-S)_{\mathrm{far}}\left(t_{1}\right)}{(I-S)_{\mathrm{far}}\left(t_{2}\right)}\right)=\ln \left(\frac{\left(I_{0}-S\right)_{\mathrm{far}}\left(t_{1}\right) e^{\tau_{1} \mathrm{far}}}{\left(I_{0}-S\right)_{\mathrm{far}}\left(t_{2}\right) e^{\tau_{2} \mathrm{far}}}\right) \\
& \quad=\left(\tau_{\mathrm{far}}\left(t_{2}\right)-\tau_{\mathrm{far}}\left(t_{1}\right)\right) .
\end{aligned}
$$

A problem with Eq. (A4) is that the auto focus of the camera causes each picture to have somewhat different brightness and contrast. This means we can't take the direct ratio of two different images without calibrating in some sense. To 
Table A1. Summary of changes in optical depth at visible wavelengths and $197 \mathrm{GHz}$.

\begin{tabular}{llclccl}
\hline Picture (time) & $\begin{array}{l}\text { Far } \\
\text { Location }\end{array}$ & $\begin{array}{l}\text { Medium } \\
\text { Location }\end{array}$ & $\begin{array}{l}\text { Near } \\
\text { Location }\end{array}$ & $\begin{array}{c}S \\
\text { value }\end{array}$ & $197.5 \mathrm{GHz}$ & $\begin{array}{l}197.5 \mathrm{GHz} \\
(10 \mathrm{~s} \mathrm{avg})\end{array}$ \\
\hline $3(14: 52: 23)$ & 0.586 & 0.0407 & 0.013 & 215 & 0.5983 & 0.6355 \\
$4(14: 52: 32)$ & 0.76 & 0.0573 & 0.0362 & 220 & 0.5324 & 0.6463 \\
$5(14: 53: 09)$ & 1.1173 & 0.1139 & 0.0565 & 225 & 0.8698 & 0.822 \\
$6(15: 03: 36)$ & 2.8736 & 0.4451 & 0.2426 & 210 & 1.8558 & 1.8384 \\
$7(15: 10: 14)$ & 2.3435 & 0.9113 & 0.2795 & 205 & 1.9895 & 2.0105 \\
$8(15: 20: 28)$ & 2.7119 & 2.0569 & 1.5084 & 230 & 3.3322 & 3.3073 \\
\hline
\end{tabular}

get around this problem, we use the same calibration location for both pictures. By first taking the ratio of the far location to the calibration location for each picture we can eliminate camera variations as shown in Eq. (A5)

$$
\begin{aligned}
& \ln \left(\frac{\frac{(I-S)_{\mathrm{far}}\left(t_{1}\right)}{(I-S)_{\mathrm{cal}}\left(t_{1}\right)}}{\frac{(I-S)_{\mathrm{far}}\left(t_{2}\right)}{\left(I-S_{\mathrm{cal}}\left(t_{2}\right)\right)}}\right) \\
& =\ln \left(\frac{\left(I_{0}-S\right)_{\mathrm{far}}\left(t_{1}\right) e^{-\tau_{1 \mathrm{far}}}}{\left(I_{0}-S\right)_{\mathrm{far}}\left(t_{2}\right) e^{-\tau_{2} \mathrm{far}}} \frac{\left(I_{0}-S\right)_{\mathrm{cal}}\left(t_{2}\right) e^{-\tau_{1 \mathrm{cal}}}}{\left(I_{0}-S\right)_{\mathrm{cal}}\left(t_{1}\right) e^{-\tau_{2} \mathrm{cal}}}\right) .
\end{aligned}
$$

After simplifying by canceling the $\left(I_{0}-S\right)$ terms we get Eq. (A6), which shows we can calculate the relative change in visual optical depth between the far location and the calibration location.

$$
\begin{aligned}
& \ln \left(\frac{\frac{(I-S)_{\mathrm{far}}\left(t_{1}\right)}{(I-S)_{\mathrm{cal}}\left(t_{1}\right)}}{\frac{(I-S)_{\mathrm{far}}\left(t_{2}\right)}{(I-S)_{\mathrm{cal}}\left(t_{2}\right)}}\right) \\
& =\left(\tau_{\mathrm{far}}\left(t_{2}\right)-\tau_{\mathrm{far}}\left(t_{1}\right)+\tau_{\mathrm{cal}}\left(t_{1}\right)-\tau_{\mathrm{cal}}\left(t_{2}\right)\right)=\Delta \tau_{\mathrm{far}}-\Delta \tau_{\mathrm{cal}} .
\end{aligned}
$$

The derivation works for the medium and near location simulations as well.

\section{A1 Results}

Figure 19 was used as the calibration or normalization picture to which all the following pictures visual optical depths were calculated from. In terms of Eq. (A6), the term, $\left(\frac{(I-S) \operatorname{far}\left(t_{1}\right)}{(I-S)_{\text {cal }}\left(t_{1}\right)}\right)$ was calculated using Fig. 19, whereas the term, $\left(\frac{(I-S)_{\mathrm{far}}\left(t_{2}\right)}{(I-S)_{\mathrm{cal}}\left(t_{2}\right)}\right)$ was calculated using the subsequent photographs so we could determine how the optical depth increased with time. Table A1 shows the relative change in optical depth for the far, medium and near locations.

Acknowledgements. This research has been funded by the National Science Foundation under grants 0723239, 0958556 and 0946411. We also greatfully acknowledge Greg Barron-Gafford at the Sustainability of semi-Arid Hydrological and Riperian Areas (SAHRA) and Russ Scott at the US Department of Agriculture USDA for providing us with access to data from their instruments in the several field experiments.

\section{References}

Anthes, R. and Committee on Earth Science and Applications from Space: A Community Assessment and Strategy for the Future, National Research Council: Earth Science and Applications from Space: National Imperatives for the Next Decade and Beyond, Space Studies Board, The National Acamdemies Press, Washington, D.C., available at: http://www.nap.edu/catalog. php?record_id=11820 (last access: December 2011), 2007.

Anthes, R. A.: Exploring Earth's atmosphere with radio occultation: contributions to weather, climate and space weather, Atmos. Meas. Tech., 4, 1077-1103, doi:10.5194/amt-4-1077-2011, 2011.

Atlas, D., Srivastava, R. S., and Sekhon, R. S.: Doppler radar characteristics of precipitation at vertical incidence, Rev. Geophys., 11, 1-35, 1973.

Bohren, C. F. and Huffman, S. R.: Absorption and Scattering of Light by Small Particles, John Wiley \& Sons, New York, 1983.

Cardinali, C.: Forecast sensitivity to observation (FSO) as a diagnostic tool, European Center for Medium-range Weather Forecasting (ECMWF) Technical Memorandum 599, October 2009, available at: http://www.ecmwf.int/publications/ (last access: December 2011), 2009.

Fraile, R. and Fernandez-Raga, M.: On a more consistent definition of radar reflectivity, Atmósfera, 22, 375-385, 2009.

Fulton, R. A., Breidenbach, J. P., Seo, D. J., and Miller, D. A.: TheWSR-88D rainfall algorithm, Weather Forecast., 13, 377395, 1998.

Gunn, R. and Kinzer, G. D.: The terminal velocity of fall for water droplets in stagnant air, J. Meteorol., 6, 243-248, 1949.

Ho, S.-P., Kirchengast, G., Leroy, S., Wickert, J., Mannucci, A. J., Steiner, A. K., Hunt, D., Schreiner, W., Sokolovskiy, S., Ao, C. O., Borsche, M., von Engeln, A., Foelsche, U., Heise, S., Iijima, B., Kuo, Y.-H., Kursinski, E. R., Pirscher, B., Ringer, M., Rocken, C., and Schmidt, T.: Estimating the Uncertainty of using GPS Radio Occultation Data for Climate Monitoring: Inter-comparison of CHAMP Refractivity Climate Records 2002-2006 from Different Data Centers, J. Geophys. Res., 114, D23107, doi:10.1029/2009JD011969, 2009.

John, V. O., Holl, G., Allan, R. P., Buehler, S. A., Parker, D. E. and Soden, B. J.: Clear-sky biases in satellite infrared estimates of upper tropospheric humidity and its trends, J. Geophys. Res., 116, D14108, doi:10.1029/2010JD015355, 2011. 
Kursinski, E. R., Hajj, G. A., Schofield, J. T., Linfield, R. P., and Hardy, K. R.: Observing Earth's atmosphere with radio occultation measurements using the Global Positioning System, J. Geophys. Res., 102, 23429-23465, 1997.

Kursinski, E. R., Feng, D., Flittner, D., Hajj, G., Herman, B., Syndergaard, S., Ward, D., and Yunck, T.: A microwave occultation observing system optimized to characterize atmospheric water, temperature and geopotential via absorption, J. Atmos. Ocean. Tech., 19, 1897-1914, 2002.

Kursinski, E. R., Feng, D., Flittner, D., Hajj, G., Herman, B., Romberg, F., Syndergaard, S., Ward, D., and Yunck, T.: An Active Microwave Limb Sounder for Profiling Water Vapor, Ozone, Temperature, Geopotential, Clouds, Isotopes and Stratospheric Winds, in: Occultations for Probing Atmosphere and Climate (OPAC-1), edited by: Kirchengast, G., Foelsche, U., and Steiner, A. K., Springer-Verlag, Berlin, 173-188, 2004.

Kursinski, E. R., Ward, D., Otarola, A., Frehlich, R., Groppi, C., Albanna, S., Schein, M., Bertiger, W., and Ross, M.: The Active Temperature Ozone and Moisture Microwave Spectrometer (ATOMMS), in: New Horizons in Occultation Research, Studies in Atmosphere and Climate, edited by: Steiner, A., Pirscher, B., Foelsche, U., and Kirchengast, G., ISBN: 978-3-642-00320-2, Hardcover, p. 336, 2009.

Lanzante, J. R. and Gahrs, G. E.: The clear-sky bias of TOVS uppertropospheric humidity, J. Climate, 13, 4034-4041, 2000.

Liebe, H. J., Hufford, G. A., and Cotton, M. G.: Propagation modeling of moist air and suspended water/ice particles at frequencies below $1000 \mathrm{GHz}$, Proc. NATO/AGARD Wave Propagation Panel, 52nd Meeting, No. 3/1-10, Mallorca, Spain, 1720 May 1993.

Marshall, J. S. and Palmer, W. M.: The distribution of raindrops with size, J. Meteorol., 5, 165-166, 1948.

Morin, E., Maddox, R. A., Goodrich, D. C. and Sorooshian, S.: Radar Z-R Relationship for Summer Monsoon Storms in Arizona, Weather Forecast., 20, 672-679, 2005.
Ochou, A. D., Zahiri, E. P., Bamba, B., and Koffi, M.: Understanding the Variability of Z-R Relationships Caused by Natural Variations in Raindrop Size Distributions (DSD): Implication of Drop Size and Number, available at: http:// www.SciRP.org/journal/acs/, Atmos. Clim. Sci., 1, 147-164, doi:10.4236/acs.2011.13017, 2011.

Paine, S.: Atmospheric Model am 6.2, available at: http://www. cfa.harvard.edu/ $\backslash$ simspaine/am/, last access: December 2011 , Harvard-Smithsonian Center for Astrophysics, Cambridge, MA, 02138, 2011.

Payne, V. H., Delamere, J. S., Cady-Pereira, K. E., Gamache, R. R., Moncet, J.-L., Mlawer, E. J., and Clough, S. A.: Air-Broadened Half-Widths of the 22- and 183-GHz Water-Vapor Lines, IEEE T. Geosci. Remote, 46, 3601-3617, 2008.

Rossow, W. B. and Schiffer, R. A.: Advances in understanding clouds from the ISCCP, B. Am. Meteorol. Soc., 80, 2261-2287, 1999.

Waters, J. W., Froidevaux, L., Harwood, R. S., Jarnot, R. F., Pickett, H. M., Read, W. G., Siegel, P. H., Cofield, R. E., Filipiak, M. J., Flower, D. A., Holden, J. R., Lau, G. K., Livesey, N. J., Manney, G. L., Pumphrey, H. C., Santee, M. L., Wu, D. L., Cuddy, D. T., Lay, R. R., Loo, M. S., Perun, V. S., Schwartz, M. J., Stek, P. C., Thurstans, R. P., Boyles, M. A., Chandra, K. M., Chavez, M. C., Chen, G.-S., Chudasama, B. V., Dodge, R., Fuller, R. A., Girard, M. A., Jiang, J. H., Jiang, Y., Knosp, B. W., LaBelle, R. C., Lam, J. C., Lee, K. A., Miller, D., Oswald, J. E., Patel, N. C., Pukala, D. M., Quintero, O., Scaff, D. M., Van Snyder, W., Tope, M. C., Wagner, P. A., and Walch, M. J.: The Earth Observing System Microwave Limb Sounder (EOS MLS) on the Aura Satellite, IEEE T. Geosci. Remote, 44, 1075-1092, 2006.

Williams, C. R. and Gage, K. S.: Raindrop size distribution variability estimated using ensemble statistics, Ann. Geophys., 27, 555-567, doi:10.5194/angeo-27-555-2009, 2009. 